\title{
An accurate discretization for an \\ inhomogeneous transport equation with arbitrary coefficients and source
}

\author{
Antonio Pascau ${ }^{\dagger}$, Muslum Arici ${ }^{\ddagger}$ \\ $\dagger$ Fluid Mechanics Group and LIFTEC, CSIC-University of Zaragoza, María de \\ Luna 3, 50018 Zaragoza, Spain \\ $\ddagger$ Kocaeli University, Engineering Faculty, Mechanical Engineering Department, \\ Umuttepe Campus, 41380 Kocaeli, Turkey
}

\begin{abstract}
A new way of obtaining the algebraic relation between the nodal values in a general onedimensional transport equation is presented. The equation can contain an arbitrary source and both the convective flux and the diffusion coefficient may vary arbitrarily. Contrary to the usual approach of approximating the derivatives involved, the algebraic relation is based on the exact solution written in integral terms. The required integrals can be speedily evaluated by approximating the integrand with Hermite splines or applying Gauss quadrature rules. The startling point about the whole procedure is that a very high accuracy can be obtained with few nodes, and more surprisingly, it can be increased almost up to machine accuracy by augmenting the number of quadrature points or the Hermite spline degree with little extra cost.
\end{abstract}

Key words: CFD, Nonhomogeneous transport equation, Convection Diffusion, Integral solution

PACS: 47.11.+j 


\section{Introduction}

Transport equations are partial differential equations (PDE) that are ubiquitous in many branches of science, in particular Fluid Mechanics. They govern the evolution of flow variables whose values, for one reason or another, are required to be known in a certain domain. Unfortunately, an analytical solution to these equations is seldom possible, so in order to know the field at a discrete number of points one has to resort to numerical techniques that provide an approximate solution. The computational techniques employed for the fluid mechanics equations gave birth to a branch called Computational Fluid Dynamics (CFD) that nowadays has almost constituted a separate subject.

There has been a huge effort along the years to improve the algorithms devised to obtain the flow field solutions with general numerical methods: finite differences, elements, volumes or spectral. Finite differences and volumes employ a numerical approximation to the derivatives present in the equation, whereas, generally speaking, finite elements or spectral techniques use a kind of solution expansion either in a local or global basis. Usually these approaches are onedimensional: the discretization along one coordinate is independent of the others. For a standard discretization it is worth pointing out that none of these methods use the solution of the ordinary differential equation (ODE) that can be obtained if the multidimensional partial differential equation (PDE)

\footnotetext{
Email addresses: pascau@unizar.es (Antonio Pascau),
} muslumarici@gmail.com (Muslum Arici). 
is integrated over an interval along a given coordinate. As a result of this integration the PDE converts into an nonhomogeneous first-order ODE whose solution can be written in terms of its homogeneous and particular solutions via the general theory of first-order ODEs. The method proposed in this work uses the exact integral solution of the first-order ODE to obtain the algebraic nodal equations of the second-order PDE, and it is different in that sense from previous methods. The examples presented in this paper are however limited to onedimensional convection diffusion problems, that is, second-order ODEs.

There have been several attempts to use the exact solution of a transport equation in the derivation of the algebraic coefficients. The pioneering paper is that of Raithby at al. [1] in which they assessed the sources of errors in their 2D discretization by comparing it with the local unidirectional exact solution in which all cross-stream fluxes were lumped together into a pseudo-source. This source was constant in the interval between two consecutive nodes and the coefficients at the interface prevailed over the whole interval length. Based on this they proposed LOADS (Locally Analytic Differencing Scheme) where they made the exact equation to match the values at two consecutive nodes, thereby obtaining the numerical fluxes for each face of the control volume and, by summing up the fluxes, an algebraic equation for every node. This scheme basically was a conservative extension of the Allen and Southwell scheme [2] which was nonconservative. Thiart [3] [4] used a collocated grid to implement the same idea for the Navier-Stokes equations. In the first paper only the external source was considered to form part of the exact solution but in the second he also included the cross-stream terms in the modified source. In these two papers the source was constant in every subinterval that belonged to a control volume and discontinuous at the interfaces. Harms et al. [5] and later 
Wang et al [6] extended this scheme to interfaces not located midway between two consecutive nodes as Thiart's scheme required.

In the early 90's two schemes that used the exact solution were LECUSSO (Locally exact consistent upwind scheme of second order) and LENS (Locally exact numerical scheme) [8] [7]. The second one can deal with a wider range of problems because the exact solution for a constant-coefficient, linear absorption, polynomial-source ODE was employed in its derivation. When the absorption is zero and the source is constant LENS transforms into LECUSSO. All algebraic coefficients are obtained by adjusting the exact solution over five nodes. Later Sakai put forward an optimized version of both [9] [10]. A final improvement of LENS was to incorporate four different zones of piecewiseconstant diffusion and absorption coefficients within a three-node region [11]. A linearly varying diffusion and absorption coefficients were also considered by Kriventsev et al. [12].

In order to mimic the exact solution a set of methods used a test function inside the control volume that contained a sum of three terms: a constant, an exponential of the Péclet number based on a local coordinate $x$ and a linear term of the same. The associated constants were determined by requiring the function to pass through the nodal values. All of them were logical inhomogeneous extensions of the exponential scheme which is known to be exact in 1D with constant coefficients and no source. The third term appeared because the exact solution with a constant source in the control volume contains a linear term related to the source. Amongst these approaches is the UNIFAES scheme [13] [14] and the scheme adopted by Sheu et al. [15]. In the latter a linear absorption term was also included. The UNIFAES was again based in Allen and Southwell scheme with the constant in the source-related term 
being linearly interpolated at the interface from its values at the nodes, these latter obtained following Allen and Southwell idea. This interpolation makes the whole scheme conservative yet it is based in a nonconservative one.

Because they have sparked lines of research of their own it is adequate to comment apart on two general approaches that employ in one way or another the exact solution: the finite analytic method (FA) [16] [17] and the nodal integral method (NIM) [19] [20]. The main idea of the FA method is applicable to any unsteady multidimensional transport equation. A local domain is considered around a generic node P. For any spatial boundary the method assumes that the solution contains the same three terms as before as well as a linear time dependence in the temporal boundaries. These boundary conditions are written in terms of the boundary nodes (those surrounding P). Applying separation of variables one is able to obtain the exact solution in the local domain. With this solution the coefficients that should multiply the boundary values to obtain the value at $\mathrm{P}$ can be obtained. For a detailed description and many applications of the FA method see [18]. NIM, on the other hand, uses the exact solution with constant coefficients to derive the solution of the variable obtained by line-averaging the original equation around $\mathrm{P}$, either spatial or temporal. For instance, in a 1D spatial domain NIM integrates the variable over the spatial or the temporal coordinate producing two ODEs, one for the mean spatial value around $\mathrm{P}$ and other for the mean temporal value. All terms with derivatives with respect to the other coordinates are lumped into a pseudo-source term. The integration of this pseudo-source is performed by Legendre polynomials truncated at the desired degree. NIM then uses the exact solution to obtain that of these two first-order ODE, written in terms of the variable at the nodes. By algebraically manipulating this expressions and 
applying continuity constraints NIM is able to derive two coupled algebraic equations for both nodal means with a three-node stencil.

As a resumé, almost all attempts to use the exact solution of a nonhomogeneous convection-diffusion equation as a base for discretization schemes have been with constant coefficients and very simple polynomial sources. In this short review the only schemes that employ a varying diffusion coefficient are those of Sakai et al. [11] and Kriventsev et al. [12]. None of them considered varying convective flux even though in $2 \mathrm{D}$ or $3 \mathrm{D}$ the mass flux varies along a coordinate even if the divergence of the mass flux is zero.

In a former paper the first author developed a scheme named ENATE for a transport equation with constant coefficients that can handle arbitrary sources as long as they have continuous derivatives of any order in the working interval [22]. In this paper the exact integral solution of the transport equation is employed to extend this idea to arbitrary coefficients. The idea followed in this paper is very close to that proposed by ten Thije Boonkamp and Anthonissen [21] in its FV-CF scheme (Finite volume-complete flux). They look for an integral representation of the homogeneous and inhomogeneous fluxes at control volume faces of a general steady conservation law in terms of the nodes that share the face. It can be checked that the integrals involved in both fluxes are the same as those that can be derived from the approach presented in this paper, apart from the very different nomenclature employed and the path taken for its derivation. It could not be otherwise as the solution of an inhomogeneous ODE with given boundary conditions is unique. The main differences with this paper is that they work with fluxes at the faces and we work with the exact solution between nodes that allows us to present the scheme in terms of an algebraic equation with three nodes. The coefficients of this algebraic equa- 
tion are clearly defined in terms of integrals between nodes which facilitates their coding. On top of that, when it comes to computing the several cases presented, ten Thije Boonkamp and Anthonissen assume linear dependencies of the integrand, that is, the standard trapezoidal rule for integral evaluation, which overly reduces their accuracy.

This paper is structured as follows: firstly, the integral solution to a homogeneous transport equation will be derived. Then, how to deal with the source in a nonhomogeneous equation will be described. As the complete solution is the sum of the homogeneous and the particular solutions, the latter in integral terms will eventually be obtained in this section. The complete solution will then be employed to obtain the algebraic connections between nodes and the extra terms due to the source, with some discussion on the asymptotic regime of mesh Péclet going to infinity. The accuracy of the discretization is connected to a numerical integration problem and some integration alternatives employed in this paper will then be described. Finally the approach is applied to three test cases with spatially varying convective flux, diffusion coefficient and/or source, showing its excellent behaviour.

\section{Integral solution of a 1D homogeneous transport equation}

The nonhomogeneous convection-diffusion equation with variable coefficients can be written as

$$
\frac{d}{d x}\left(\rho v \phi-\Gamma \frac{d \phi}{d x}\right)=S \quad ; \quad \rho v=\rho v(x) \quad ; \quad \Gamma=\Gamma(x) \quad ; \quad S=S(x)
$$


In this section we will derive the integral solution of the homogeneous equation, $S=0$, and its simplified variants, some of them well known. This integral solution is later employed as a constituent part of the solution of the general nonhomogeneous equation with arbitrary source. As will later be seen the homogeneous solution partly contributes to the coefficients that connect nodal values in the final algebraic equation.

The domain is split in $N$ intervals, not necessarily of equal length, and $N+1$ nodes with locations $x_{i}, i=0, \ldots, N$, with two nodes at the boundaries, $x_{0}$ and $x_{N}$. In order to obtain the homogeneous solution in every generic interval with left boundary $(l b)$ and right boundary $(r b)$, it is more convenient to work with normalized variables, defined as

$$
\widehat{\phi}=\frac{\phi-\phi_{l b}}{\phi_{r b}-\phi_{l b}}=\frac{\phi-\phi_{l b}}{\Delta \phi} \quad ; \quad \widehat{\rho v}=\frac{\rho v}{(\rho v)_{l b}} \quad ; \quad \widehat{\Gamma}=\frac{\Gamma}{\Gamma_{l b}} \quad ; \quad \widehat{\lambda}=\frac{\widehat{\rho v}}{\widehat{\Gamma}}
$$

where the values at the left boundary of the interval have been used as normalizing factors. A normalized coordinate $\widehat{x}$ is chosen as independent variable by considering a mapping between the working interval of length $L=x_{r b}-x_{l b}$, and a unity domain, $x=x_{l b}+L \widehat{x}, 0 \leqslant \widehat{x} \leqslant 1, x \in\left[x_{l b}, x_{r b}\right]$. Then, the convection diffusion equation transforms to

$$
\frac{d}{d \widehat{x}}\left(\widehat{\rho v} \widehat{\phi}-\frac{\widehat{\Gamma}}{P_{L 0}} \frac{d \widehat{\phi}}{d \widehat{x}}\right)=-\frac{\phi_{l b}}{\Delta \phi} \frac{d}{d \widehat{x}} \widehat{\rho v} \quad ; \quad P_{L 0}=\frac{(\rho v)_{l b} L}{\Gamma_{l b}}
$$

All normalized coefficients will now depend on $\widehat{x} . \phi_{l b} / \Delta \phi$ is an unknown constant for a given interval, different for each one. $P_{L 0}$ is the reference Péclet number of the interval. Let us integrate between 0 and $\widehat{x}$

$$
\widehat{\rho v} \widehat{\phi}-(\widehat{\rho v} \widehat{\phi})_{0}-\frac{\widehat{\Gamma}}{P_{L 0}} \frac{d \widehat{\phi}}{d \widehat{x}}+\left(\frac{\widehat{\Gamma}}{P_{L 0}} \frac{d \widehat{\phi}}{d \widehat{x}}\right)_{0}=-\frac{\phi_{l b}}{\Delta \phi}(\widehat{\rho v}-1)
$$


This expression is just an ordinary differential equation that contains unknown factors. Some of them can be easily determined with the boundary conditions for $\widehat{\phi}$. As $\widehat{\phi}(0)=0$, the differential equation to be solved is

$$
\frac{d \widehat{\phi}}{d \widehat{x}}-P_{L} \widehat{\phi}=P_{L 0} \frac{\phi_{l b}}{\Delta \phi} \widehat{\lambda}+\frac{1}{\widehat{\Gamma}}\left[\left(\frac{d \widehat{\phi}}{d \widehat{x}}\right)_{0}-P_{L 0} \frac{\phi_{l b}}{\Delta \phi}\right] \quad ; \quad P_{L}=\frac{\rho v L}{\Gamma}
$$

The RHS contains an a priori unknown source that depends on $\widehat{x}$ through $\hat{\lambda}$ and $\widehat{\Gamma}$. According to the theory of ODE the solution to this equation is

$$
\frac{\widehat{\phi}(\widehat{x})}{E(\widehat{x})}=\left[\left(\frac{d \widehat{\phi}}{d \widehat{x}}\right)_{0}-P_{L 0} \frac{\phi_{l b}}{\Delta \phi}\right] \int^{\widehat{x}} \frac{d \widehat{x}^{\prime}}{\widehat{\Gamma} E}+P_{L 0} \frac{\phi_{l b}}{\Delta \phi} \int^{\widehat{x}} \frac{\widehat{\lambda}}{E} d \widehat{x}^{\prime}+C_{o}
$$

being $E(\widehat{x})=\exp \int^{\widehat{x}} P_{L} d \widehat{x}^{\prime}$ the integrating factor. $C_{o}$ is an integration constant and $\widehat{x}^{\prime}$ is a dummy variable. As $\widehat{\phi}(0)=0$ we can obtain $C_{o}$ and rewrite the solution as

$$
\frac{\widehat{\phi}(\widehat{x})}{E(\widehat{x})}=\left[\left(\frac{d \widehat{\phi}}{d \widehat{x}}\right)_{0}-P_{L 0} \frac{\phi_{l b}}{\Delta \phi}\right] \int_{0}^{\widehat{x}} \frac{d \widehat{x}^{\prime}}{\widehat{\Gamma} E}+P_{L 0} \frac{\phi_{l b}}{\Delta \phi} \int_{0}^{\widehat{x}} \frac{\widehat{\lambda}}{E} d \widehat{x}^{\prime}
$$

The second boundary condition, $\widehat{\phi}(1)=1$, is employed to obtain the final solution as

$$
\begin{aligned}
\widehat{\phi}(\widehat{x}) & =\bar{E}(\widehat{x})\left(\int_{0}^{\widehat{x}} \frac{d \widehat{x}^{\prime}}{\widehat{\Gamma} \bar{E}} / \int_{0}^{1} \frac{d \widehat{x}^{\prime}}{\widehat{\Gamma} \bar{E}}+\right. \\
& \left.+P_{L 0} \frac{\phi_{l b}}{\Delta \phi} \int_{0}^{1} \frac{\widehat{\lambda}}{\bar{E}} d \widehat{x}^{\prime}\left[\int_{0}^{\widehat{x}} \frac{\hat{\lambda}}{\bar{E}} d \widehat{x}^{\prime} / \int_{0}^{1} \frac{\widehat{\lambda}}{\bar{E}} d \widehat{x}^{\prime}-\int_{0}^{\widehat{x}} \frac{d \widehat{x}^{\prime}}{\widehat{\Gamma} \bar{E}} / \int_{0}^{1} \frac{d \widehat{x}^{\prime}}{\widehat{\Gamma} \bar{E}}\right]\right)
\end{aligned}
$$

where $\bar{E}$ is defined as

$$
\bar{E}(\widehat{x})=\frac{E(\widehat{x})}{E(1)}=\exp \left(\int^{\widehat{x}} P_{L} d \widehat{x}^{\prime}\right) \exp \left(-\int^{1} P_{L} d \widehat{x}^{\prime}\right)=\exp \left(-\int_{\widehat{x}}^{1} P_{L} d \widehat{x}^{\prime}\right)(9)
$$


Once solved, it is straightforward to recover the values of the dimensional variable as $\phi=\phi_{l b}+\Delta \phi \widehat{\phi}$. This is the most general solution of the homogeneous convection-diffusion equation for arbitrary coefficients. It only involves the modified integrating factor $\bar{E}$ and two other integrals. If there was only one interval, i.e., the whole domain, $\phi_{l b}$ and $\Delta \phi$ would be known if Dirichlet boundary conditions were given. This expression would then be useful for obtaining a solution if the integrals could be evaluated exactly, but in many instances this will not be the case. As some kind of polynomial approximation will be needed for the integrands, it will become necessary to split the domain in intervals of small size for which the interpolants are more accurate and where $\phi_{l b}$ and $\Delta \phi$ are unknown. In the process of discretization all interval solutions will be linked to obtain the system of equations that will provide the solution over the whole domain.

There are some particular solutions for constant $\rho v$ that are well known. If $\rho v$ is constant $\widehat{\rho v}=1$ and the two factors inside the square brackets are the same because $\widehat{\lambda}=\widehat{\rho v} / \widehat{\Gamma}=1 / \widehat{\Gamma}$. The solution is then

$$
\widehat{\phi}(\widehat{x})=\bar{E}(\widehat{x})\left(\int_{0}^{\widehat{x}} \frac{d \widehat{x}^{\prime}}{\widehat{\Gamma} \bar{E}} / \int_{0}^{1} \frac{d \widehat{x}^{\prime}}{\widehat{\Gamma} \bar{E}}\right)=\bar{E}(\widehat{x})\left(\int_{0}^{\widehat{x}} \frac{\widehat{\lambda}}{\bar{E}} d \widehat{x}^{\prime} / \int_{0}^{1} \frac{\widehat{\lambda}}{\bar{E}} d \widehat{x}^{\prime}\right)
$$

As $\widehat{\lambda} / \bar{E}=k d(1 / \bar{E}) / d \widehat{x}$ with $k=-1 / P_{L 0}$, the final solution is

$$
\widehat{\phi}(\widehat{x})=\bar{E}(\widehat{x}) \frac{1 / \bar{E}(0)-1 / \bar{E}(\widehat{x})}{1 / \bar{E}(0)-1 / \bar{E}(1)}=\frac{\exp \int_{0}^{\widehat{x}} P_{L} d \widehat{x}^{\prime}-1}{\exp \int_{0}^{1} P_{L} d \widehat{x}^{\prime}-1}
$$

Beginning with this expression there is another simplification when the Péclet number is small. In that case the exponential of the integral can be substituted by the first two terms of its Taylor series expansion and the solution only depends on the integral of $1 / \Gamma$. 


$$
\widehat{\phi}(\widehat{x})=\frac{\int_{0}^{\widehat{x}} P_{L} d \widehat{x}^{\prime}}{\int_{0}^{1} P_{L} d \widehat{x}^{\prime}}=\frac{\int_{0}^{x} \frac{d \widehat{x}^{\prime}}{\Gamma}}{\int_{0}^{1} \frac{d \widehat{x}^{\prime}}{\Gamma}}
$$

In this latter case the constant (and small) convection term does not affect the solution which corresponds to that of the diffusion equation with variable diffusivity. Both simplified expressions above are solutions of the homogeneous equation with variable $\Gamma$ and constant $\rho v$, they are particular cases of the general solution, Eqn. 8.

\section{Exact solution for arbitrary source}

We will apply the same normalization to Eqn. 1 in order to obtain the normalized equation with source

$$
\frac{d}{d \widehat{x}}\left(\widehat{\rho v} \widehat{\phi}-\frac{\widehat{\Gamma}}{P_{L 0}} \frac{d \widehat{\phi}}{d \widehat{x}}\right)=-\frac{\phi_{l b}}{\Delta \phi} \frac{d}{d \widehat{x}} \widehat{\rho v}+\Pi_{s} \quad \text { with } \quad \Pi_{s}=\frac{S(\widehat{x}) L}{(\rho v)_{l b} \Delta \phi}
$$

The new factor is $\Pi_{s}$ which is a nondimensional source. In this section we will show how to get rid of this source and eventually obtain a source-free equation. We will closely follow the derivation presented in [22] for constant coefficients. The approach is named ENATE, Enhanced Numerical Approximation of a Transport Equation, to which the reader is referred for details. The final idea is to use the solution derived in the previous section for this source-free equation.

A function $\Lambda_{s}^{(0)}$ is sought that satisfies

$$
\frac{d}{d \widehat{x}}\left(\widehat{\rho v} \Lambda_{s}^{(0)}\right)=\Pi_{s} \Rightarrow \Lambda_{s}^{(0)}=\frac{1}{\widehat{\rho v}} \int_{0}^{\widehat{x}} \Pi_{s}\left(\widehat{x}^{\prime}\right) d \widehat{x}^{\prime}
$$

which forms part of a new variable $\widetilde{\phi}=\widehat{\phi}-\Lambda_{s}^{(0)}$. The transport equation for this new variable is 


$$
\frac{d}{d \widehat{x}}\left(\widehat{\rho v} \tilde{\phi}-\frac{\widehat{\Gamma}}{P_{L 0}} \frac{d \widetilde{\phi}}{d \widehat{x}}\right)=-\frac{\phi_{l b}}{\Delta \phi} \frac{d}{d \widetilde{x}} \widehat{\rho v}+\frac{d}{d \widehat{x}}\left(\frac{\widehat{\Gamma}}{P_{L 0}} \frac{d \Lambda_{s}^{(0)}}{d \widehat{x}}\right)
$$

We can proceed in the same fashion by looking for another variable such that its first derivative is the new source

$$
\begin{aligned}
& \frac{d}{d \widehat{x}}\left(\widehat{\rho v} \Lambda_{s}^{(1)}\right)=\frac{1}{P_{L 0}} \frac{d}{d \widehat{x}}\left(\widehat{\Gamma} \frac{d \Lambda_{s}^{(0)}}{d \widehat{x}}\right) \Rightarrow \\
& \widehat{\rho v} \Lambda_{s}^{(1)}=\frac{1}{P_{L 0}}\left(\widehat{\Gamma} \frac{d \Lambda_{s}^{(0)}}{d \widehat{x}}-\left(\frac{d \Lambda_{s}^{(0)}}{d \widehat{x}}\right)_{0}\right)
\end{aligned}
$$

as $\widehat{\Gamma}(0)=1$. Without lack of generality $\Lambda_{s}^{(0)}(0)=\Lambda_{s}^{(1)}(0)=0$ is assumed (see [22] for details). As every new variable is less than the former by a factor given by the inverse of the Péclet number, the procedure will eventually end up with a source-free equation for a well-behaved original source if the Péclet number is high. By appropriately rearranging the $\Lambda$ definition equations it will later be shown that a high Péclet is not even necessary to obtain a solution for the sum of Lambdas.

The final variable is $\bar{\phi}=\widehat{\phi}-\sum_{j=0}^{\infty} \Lambda_{s}^{(j)}$ whose transport equation no longer contains $\Pi_{s}$. The solution to this equation would be Eqn. 8 if the boundary conditions were zero and one but these are not those associated to $\bar{\phi}$. In fact, by construction all Lambdas are zero at $\widehat{x}=0$ so $\bar{\phi}(0)=\widehat{\phi}(0)-\sum_{j=0}^{\infty} \Lambda_{s}^{(j)}(0)=0$ but at $\widehat{x}=1$ the value is unknown. However, a new variable may be defined as

$$
\bar{\phi}^{N}=\frac{\bar{\phi}-\bar{\phi}(0)}{\bar{\phi}(1)-\bar{\phi}(0)}=\frac{\widehat{\phi}-\sum_{j=0}^{\infty} \Lambda_{s}^{(j)}(\widehat{x})}{1-\sum_{j=0}^{\infty} \Lambda_{s}^{(j)}(1)}=\frac{\widehat{\phi}-F(\widehat{x})}{1-F(1)} \quad ; \quad F=\sum_{j=0}^{\infty} \Lambda_{s}^{(j)}
$$

for which the boundary conditions are those mentioned. The equation that governs the transport of $\bar{\phi}^{N}$ is 


$$
\frac{d}{d \widehat{x}}\left(\widehat{\rho v} \bar{\phi}^{N}-\frac{\widehat{\Gamma}}{P_{L 0}} \frac{d \bar{\phi}^{N}}{d \widehat{x}}\right)=-\frac{1}{1-F(1)} \frac{\phi_{l b}}{\Delta \phi} \frac{d}{d \widehat{x}} \widehat{\rho v}
$$

whose solution, according to Eqn. 8, is

$$
\begin{aligned}
\bar{\phi}^{N}(\widehat{x}) & =\bar{E}(\widehat{x})\left[\int_{0}^{\widehat{x}} \frac{d \widehat{x}^{\prime}}{\widehat{\Gamma} \bar{E}} / \int_{0}^{1} \frac{d \widehat{x}^{\prime}}{\widehat{\Gamma} \bar{E}}+\right. \\
& \left.+P_{L 0} \Phi \int_{0}^{1} \frac{\widehat{\lambda}}{\bar{E}} d \widehat{x}^{\prime}\left[\int_{0}^{\widehat{x}} \frac{\hat{\lambda}}{\bar{E}} d \widehat{x}^{\prime} / \int_{0}^{1} \frac{\widehat{\lambda}}{\bar{E}} d \widehat{x}^{\prime}-\int_{0}^{\widehat{x}} \frac{d \widehat{x}^{\prime}}{\widehat{\Gamma} \bar{E}} / \int_{0}^{1} \frac{d \widehat{x}^{\prime}}{\widehat{\Gamma} \bar{E}}\right]\right] \\
\Phi & =\frac{1}{1-F(1)} \frac{\phi_{l b}}{\Delta \phi}
\end{aligned}
$$

From Eqn. 17 the complete solution is thus

$$
\widehat{\phi}=F(\widehat{x})+(1-F(1)) \bar{\phi}^{N}(\widehat{x})
$$

This expression represents the solution to the normalized equation as the sum of the particular solution $F(\widehat{x})$ and the homogeneous solution $\bar{\phi}^{N}(\widehat{x})$ affected by a weighting coefficient that allows the complete solution to satisfy the boundary conditions.

\section{Generalized differential equation for $F=\sum \Lambda_{s}^{(j)}$}

As described in a previous paragraph an important variable that comes up following the ENATE approach is the sum of the source-dependent $\Lambda$ 's. There is a direct way of obtaining this sum by deriving an ODE that contains it as unknown. Let us look at the definitions of the $\Lambda$ 's

$$
\widehat{\rho v} \Lambda_{s}^{(0)}=\int_{0}^{\widehat{x}} \Pi_{s} d \widehat{x}^{\prime}
$$




$$
\begin{aligned}
& \widehat{\rho v} \Lambda_{s}^{(1)}=\frac{1}{P_{L 0}}\left(\widehat{\Gamma} \frac{d \Lambda_{s}^{(0)}}{d \widehat{x}}-\left(\frac{d \Lambda_{s}^{(0)}}{d \widehat{x}}\right)_{0}\right) \\
& \widehat{\rho v} \Lambda_{s}^{(j)}=\frac{1}{P_{L 0}}\left(\widehat{\Gamma} \frac{d \Lambda_{s}^{(j-1)}}{d \widehat{x}}-\left(\frac{d \Lambda_{s}^{(j-1)}}{d \widehat{x}}\right)_{0}\right)
\end{aligned}
$$

All equations may be summed up to obtain

$$
\widehat{\rho v} F=\int_{0}^{\widehat{x}} \Pi_{s} d \widehat{x}^{\prime}+\frac{1}{P_{L 0}}\left(\widehat{\Gamma} \frac{d F}{d \widehat{x}}-\left(\frac{d F}{d \widehat{x}}\right)_{0}\right)
$$

This is again an ordinary differential equation with constants to be determined. Rearranging,

$$
\begin{aligned}
\frac{d F}{d \widehat{x}}-P_{L} F & =-\frac{P_{L 0}}{\widehat{\Gamma}} \int_{0}^{\widehat{x}} \Pi_{s} d \widehat{x}^{\prime}+\left(\frac{d F}{d \widehat{x}}\right)_{0} \frac{1}{\widehat{\Gamma}} \\
& =-P_{L 0} \frac{\widetilde{\Pi}_{s}}{\widehat{\Gamma}}+\left(\frac{d F}{d \widehat{x}}\right)_{0} \frac{1}{\widehat{\Gamma}} ; \quad \widetilde{\Pi}_{s}(\widehat{x})=\int_{0}^{\widehat{x}} \Pi_{s} d \widehat{x}^{\prime}
\end{aligned}
$$

There is a whole family of $F$ functions, each one related to the particular value of $(d F / d \widehat{x})_{0}$. The governing differential equation has been derived assuming that $F(0)=0$ but note that the second boundary condition for $F$, that would allow us to obtain one specific solution out of the family set, is lacking. Thus, the general solution is a one-parameter set of $F$ functions that written in terms of $F(1)$ is

$$
\begin{aligned}
F(\widehat{x}) & =\bar{E}(\widehat{x})\left[F(1) \int_{0}^{\widehat{x}} \frac{d \widehat{x}^{\prime}}{\widehat{\Gamma} \bar{E}} / \int_{0}^{1} \frac{d \widehat{x}^{\prime}}{\widehat{\Gamma} \bar{E}}\right. \\
& \left.-P_{L 0} \int_{0}^{1} \frac{\widetilde{\Pi}_{s}}{\widehat{\Gamma} \bar{E}} d \widehat{x}^{\prime}\left[\int_{0}^{\widehat{x}} \frac{\widetilde{\Pi}_{s}}{\widehat{\Gamma} \bar{E}} d \widehat{x}^{\prime} / \int_{0}^{1} \frac{\widetilde{\Pi}_{s}}{\widehat{\Gamma} \bar{E}} d \widehat{x}^{\prime}-\int_{0}^{\widehat{x}} \frac{d \widehat{x}^{\prime}}{\widehat{\Gamma} \bar{E}} / \int_{0}^{1} \frac{d \widehat{x}^{\prime}}{\widehat{\Gamma} \bar{E}}\right]\right]
\end{aligned}
$$


where $\widetilde{\Pi}_{s}\left(\widehat{x}^{\prime}\right)=\int_{0}^{\widehat{x}^{\prime}} \Pi_{s} d \widehat{x}^{\prime \prime} . F(1)$ will disappear in the final equation for $\widehat{\phi}$ so there is no need to speculate which value is appropriate. As observed there is only one new integral to be evaluated, that associated to the source.

The expression obtained is nothing but the particular solution of the nonhomogeneous equation written in integral form. The governing differential equation for $F$ has been obtained via a previous definition of the Lambda functions because this was the approach followed in a former paper by the first author but, alternatively, it could have been obtained directly from the original ODE. It just highlights the fact that the sum of Lambdas of the previous paper is the function that is the particular solution to the original ODE. It can easily be checked that $\widehat{\phi}(\widehat{x})$ of Eqn 20 satisfies the original ODE just by substituting $F(\widehat{x})$ and $\bar{\phi}^{N}(\widehat{x})$ expressions.

\section{Algebraic relation between nodal values}

The solution in an arbitrary interval is

$$
\widehat{\phi}(\widehat{x})=F(\widehat{x})+(1-F(1)) \bar{\phi}^{N}(\widehat{x})
$$

$F$ represents the particular solution to the PDE and $\bar{\phi}^{N}$ the normalized homogeneous solution. In this expression it can be observed that $1-F(1)$ acts as a weighting coefficient that allows the complete solution to satisfy the boundary conditions. For each $F$ function the weighting factor is different but the final solution for $\widehat{\phi}$ is the same whatever $F$ is chosen.

The ENATE procedure consists in calculating the diffusive flux at a generic node $P$ by considering it as the end point of one interval, $W P$, between the 
$W$ est node and $P$, or the start point of the next one, $P E$, between $P$ and the East node. The diffusive flux is

$$
\Gamma \frac{\mathrm{d} \phi}{d x}=\Gamma \frac{\Delta \phi}{L} \frac{d \widehat{\phi}}{d \widehat{x}}=\rho v \Delta \phi \frac{1}{P_{L}} \frac{d \widehat{\phi}}{d \widehat{x}}=\rho v \Delta \phi\left[\frac{1}{P_{L}} \frac{d F}{d \widehat{x}}+(1-F(1)) \frac{1}{P_{L}} \frac{d \bar{\phi}^{N}}{d \widehat{x}}\right]
$$

We are equalling the two fluxes at $\mathrm{P}$, that calculated at the end of the first interval and that calculated at the start of the second. For the diffusive flux to be continuous at $P$ the source cannot be a Dirac delta, so this is the only restriction. Even if it were, with known intensity, we could establish a similar equation by including the flux jump equal to the delta amplitude. In the last case, a node should be located at the delta position because the flux relation is applied in one node.

$$
\begin{aligned}
& (\rho v)_{P} \Delta \phi_{W P}\left[\frac{1}{P_{L_{W P}}} \frac{d F}{d \widehat{x}}+(1-F(1)) \frac{1}{P_{L_{W P}}} \frac{d \bar{\phi}^{N}}{d \widehat{x}}\right]_{\widehat{x}=1} \\
= & (\rho v)_{P} \Delta \phi_{P E}\left[\frac{1}{P_{L_{P E}}} \frac{d F}{d \widehat{x}}+(1-F(1)) \frac{1}{P_{L_{P E}}} \frac{d \bar{\phi}^{N}}{d \widehat{x}}\right]_{\widehat{x}=0}
\end{aligned}
$$

It is thus obvious that the derivatives of the two functions are required at the interval edges. The complete derivation is given in the appendix, here we will present the final expression

$$
\begin{aligned}
& {\left[(\rho v)_{W} \widetilde{k}_{W P}+(\rho v)_{P}\left(\widetilde{k}_{P E}+\left.\frac{I L E_{01}}{I G E_{01}}\right|_{P E}\right)\right] \phi_{P}=} \\
& (\rho v)_{W}\left(\widetilde{k}_{W P}+\left.\frac{I L E_{01}}{I G E_{01}}\right|_{W P}\right) \phi_{W}+(\rho v)_{P} \widetilde{k}_{P E} \phi_{E} \\
& +\left.I S_{01}\right|_{W P} \\
& +\left(\left.\frac{I S G E_{01}}{I G E_{01}}\right|_{P E}-\left.\frac{I S G E_{01}}{I G E_{01}}\right|_{W P}\right)
\end{aligned}
$$

The different factors that appear in the formulation are 


$$
\begin{aligned}
& I L E_{01}=\int_{0}^{1} \frac{\widehat{\lambda}}{\bar{E}} d \widehat{x}^{\prime} \quad ; \quad I G E_{01}=\int_{0}^{1} \frac{d \widehat{x}^{\prime}}{\widehat{\Gamma} \bar{E}} ; \quad \tilde{k}=\frac{1}{P_{L 0} I G E_{01}} \\
& I S G E_{01}=\int_{0}^{1} \frac{I S_{0 \widehat{x}^{\prime}}}{\widehat{\Gamma} \bar{E}} d \widehat{x}^{\prime}=\int_{0}^{1} \frac{L \int_{0}^{x^{\prime}} S\left(\widehat{x}^{\prime \prime}\right) d \widehat{x}^{\prime \prime}}{\widehat{\Gamma} \bar{E}} d \widehat{x}^{\prime} \\
& I S_{01}=L \int_{0}^{1} S(\widehat{x}) d \widehat{x}=\int_{x_{l b}}^{x_{r b}} S(x) d x
\end{aligned}
$$

Note that the two factors related to the source term, ISGE and IS, are dimensional, the rest are nondimensional. The algebraic equation obtained is cast in the traditional way. The only difference with standard discretizations is that the coefficients that link the variables at the nodes have not been obtained by numerically approximating the derivatives of the original ODE, but by relying on the integral solution. The expression is exact, that is, were we able to calculate the integrals exactly we would obtain the exact solution for arbitrary coefficients and source. Thus, the discretization problem has been transferred from derivatives to integrals, allowing, as will be shown, higher accuracy. As all integrals are carried out in intervals between nodes and the integrands are to be approximated with continuous functions the only restriction is that the coefficients should be continuous with continuous derivatives up to a degree depending on the degree of the approximating polynomial. When describing the interpolating polynomials we will be more specific on this restriction. Note that a coefficient and/or the source may be discontinuous (or their derivatives) but in that case a node should be placed in the discontinuity.

It was mentioned previously that this work is a generalization of a previous one concerning a convection-diffusion equation with arbitrary source. In that paper both the mass flux and the diffusion coefficient were constant in a given interval whereas in this one both are arbitrary. Obviously, if the coefficients 
are piecewise-constant in Eqn. 28 we should recover the expressions of the previous paper. In order to connect both papers a short derivation will be provided. We do not mean to be exhaustive, we will only detail the equality of the source integral term of that paper, the others follow suit with the same procedure.

Let us calculate all factors involved when the coefficients are constant over an interval. In that case $I L E_{01}=I G E_{01}$ and $I G E_{01}=\left(\exp P_{L}-1\right) / P_{L}$, so $\widetilde{k}_{W P}$ of this work is $\widetilde{k}_{1}$ of the previous one, and $1+\widetilde{k}_{P E}$ is $\widetilde{k}_{2}$. From Eqn. (A.9) we can obtain

$$
\frac{I S G E_{01}}{I G E_{01}}=(\rho v)_{l b} \Delta \phi\left(\left.\frac{1}{P_{L 0}} \frac{d F}{d \widehat{x}}\right|_{0}-\widetilde{k} F(1)\right)
$$

In the case of constant coefficients $I S G E_{01} / I G E_{01}$ is given by

$$
\frac{I S G E_{01}}{I G E_{01}}=\rho v\left[-\frac{\sum_{j=0}^{\infty} \Lambda_{s}^{(j)}(1)}{\exp P_{L}-1}+\left.\frac{1}{P_{L}} \sum_{j=0}^{\infty} \frac{d \Lambda_{s}^{(j)}}{d \widehat{x}}\right|_{0}\right]
$$

The Lambdas in this expression are those in Eqn. 21 multiplied by $\Delta \phi$ as they were in the previous paper. If we take the first term of the series, that of $\Lambda_{s}^{(0)}$ in $W P$, we have

$$
\begin{aligned}
-\left.\frac{I S G E_{01}}{I G E_{01}}\right|_{W P}+\left.L \int_{0}^{1} S(\widehat{x}) d \widehat{x}\right|_{W P} & =\frac{\rho v \Lambda_{s}^{(0)}(1)}{\exp P_{L}-1}+\rho v \Lambda_{s}^{(0)}(1) \\
& =\rho v \frac{\exp P_{L}}{\exp P_{L}-1} \Lambda_{s}^{(0)}(1) \\
& =\widetilde{k}_{1} \int_{x_{W}}^{x_{P}} S d x
\end{aligned}
$$

because by definition

$$
\widetilde{k}_{1}=\frac{\exp P_{L}}{\exp P_{L}-1} \quad ; \quad \rho v \Lambda_{s}^{(0)}(1)=L \int_{0}^{1} S(\widehat{x}) d \widehat{x}=\int_{x_{W}}^{x_{P}} S d x
$$


This term is the source integral of the final algebraic expression of the previous paper. Likewise, the first term of $I S G E / I G E$ associated to $P E$ is

$$
\left.\frac{I S G E_{01}}{I G E_{01}}\right|_{P E}=-\frac{\rho v \Lambda_{s}^{(0)}(1)}{\exp P_{L}-1}=-\widetilde{k}_{2} \int_{x_{P}}^{x_{E}} S d x \quad ; \quad \widetilde{k}_{2}=\frac{1}{\exp P_{L}-1}
$$

The rest of terms concerning the successive source derivatives connected to $\Lambda_{s}^{(j)}$ can be obtained similarly showing that both formulations are identical in the case of piecewise-constant coefficients.

In the final expression, Eqn 28, there is no explicit contribution of the convective flux at node $E$, its effect is only felt in the integrals of $\hat{\lambda}$ and $\bar{E}$. The integral that contains $\hat{\lambda}$ is exact only if the integral of $P_{L}$ is known

$$
\int_{0}^{1} \frac{\widehat{\lambda}}{\bar{E}} d \widehat{x}^{\prime}=-\frac{1}{P_{L 0}} \int_{0}^{1} \frac{d}{d \widehat{x}^{\prime}}\left(\frac{1}{\bar{E}}\right) d \widehat{x}^{\prime}=\frac{1}{P_{L 0}}\left(\exp \int_{0}^{1} P_{L} d \widehat{x}^{\prime}-1\right)
$$

For the other integrals it is worth describing several special cases associated to high mesh Péclet numbers for which the exponentials can become excessively large for them to be represented as a double precision number. For instance, $I G E_{01}$ can be approximated by

$$
\begin{aligned}
I G E_{01} & =\int_{0}^{1} \frac{d \widehat{x}^{\prime}}{\widehat{\Gamma} \bar{E}}=\int_{0}^{1} \frac{1}{\widehat{\rho v}} \frac{\widehat{\lambda}}{\bar{E}} d \widehat{x}^{\prime}=-\frac{1}{P_{L 0}} \int_{0}^{1} \frac{1}{\widehat{\rho v}} \frac{d}{d \widehat{x}^{\prime}}\left(\frac{1}{\bar{E}}\right) d \widehat{x}^{\prime} \approx \\
& \approx-\frac{1}{P_{L 0}} \int_{0}^{1} \frac{d}{d \widehat{x}^{\prime}}\left(\frac{1}{\widehat{\rho v} \bar{E}}\right) d \widehat{x}^{\prime}=\frac{1}{P_{L 0}}\left(\exp \int_{0}^{1} P_{L} d \widehat{x}^{\prime}-\frac{1}{\left.\widehat{\rho v}\right|_{1}}\right)
\end{aligned}
$$

The approximation indicated in the integral is acceptable if

$$
\left|\frac{1}{\bar{E}} \frac{d}{d \widehat{x}}\left(\frac{1}{\widehat{\rho v}}\right)\right| \ll\left|\frac{1}{\widehat{\rho v}} \frac{d}{d \widehat{x}}\left(\frac{1}{\bar{E}}\right)\right| \quad \text { or } \quad\left|\frac{d}{d x}\left(\frac{1}{v}\right)\right| \ll \frac{1}{\alpha} ; \alpha=\frac{\Gamma}{\rho}
$$

Note that the second inequality relates to dimensional variables. The region where the inequality will likely be satisfied is that of high mesh Péclet numbers. 
In that region

$$
\frac{I L E_{01}}{I G E_{01}}=\frac{\exp \int_{0}^{1} P_{L} d \widehat{x}^{\prime}-1}{\exp \int_{0}^{1} P_{L} d \widehat{x}^{\prime}-1 /\left.\widehat{\rho v}\right|_{1}} \approx 1
$$

Following the same procedure we can obtain a simplified version of $I S G E_{01}$ for high mesh Péclet if an inequality for the source, in terms of dimensional variables, is satisfied. $I S G E_{01}$ can be written as

$$
\begin{aligned}
I S G E_{01} & =\int_{0}^{1} \frac{L \int_{0}^{\widehat{x}^{\prime}} S\left(\widehat{x}^{\prime \prime}\right) d \widehat{x}^{\prime \prime}}{\widehat{\Gamma} \bar{E}} d \widehat{x}^{\prime} \\
& =\int_{0}^{1} \frac{L \int^{\widehat{x}^{\prime}} S\left(\widehat{x}^{\prime \prime}\right) d \widehat{x}^{\prime \prime}}{\widehat{\Gamma} \bar{E}} d \widehat{x}^{\prime}-I G E_{01} L \int^{0} S\left(\widehat{x}^{\prime}\right) d \widehat{x}^{\prime} \\
& \approx-\frac{1}{P_{L 0}} \int_{0}^{1} \frac{d}{d \widehat{x}^{\prime}}\left(\frac{L \int^{x^{\prime}} S\left(\widehat{x}^{\prime \prime}\right) d \widehat{x}^{\prime \prime}}{\widehat{\rho v} \bar{E}}\right) d \widehat{x}^{\prime}-I G E_{01} L \int^{0} S\left(\widehat{x}^{\prime}\right) d \widehat{x}^{\prime} \\
& =-\frac{1}{P_{L 0}} \frac{L}{\left.\widehat{\rho v}\right|_{1}} \int_{0}^{1} S(\widehat{x}) d \widehat{x}
\end{aligned}
$$

For the last equality the value of $I G E_{01}$ in Eqn. 36 has been used. The approximation is valid if the following is true

$$
\left|\frac{1}{\bar{E}} \frac{d}{d \widehat{x}}\left(\frac{\int^{\widehat{x}} S\left(\widehat{x}^{\prime}\right) d \widehat{x}^{\prime}}{\widehat{\rho v}}\right)\right| \ll\left|\frac{\int^{\widehat{x}} S\left(\widehat{x}^{\prime}\right) d \widehat{x}^{\prime}}{\widehat{\rho v}} \frac{d}{d \widehat{x}}\left(\frac{1}{\bar{E}}\right)\right|
$$

or in terms of dimensional variables

$$
\left|\frac{d}{d x}\left(\frac{\int^{x} S(x) d x}{v}\right)\right| \ll \frac{\left|\int^{x} S(x) d x\right|}{\alpha}
$$

We have already assumed that the inequality in Eqn 37 is true so the additional constraint is

$$
\left|\frac{S(x)}{v}\right| \ll \frac{\left|\int^{x} S(x) d x\right|}{\alpha} \Rightarrow \frac{1}{|\lambda|} \ll l_{S} \quad ; \quad l_{S}=\frac{1}{|S(x)|}\left|\int S\left(x^{\prime}\right) d x^{\prime}\right|
$$


$l_{S}$ is a length scale related to the primitive of the source. To ascertain the behaviour of $I S G E_{01}$ when mesh Péclet is very high, let us calculate the ratio $I S G E_{01} / I G E_{01}$ in that region

$$
\frac{I S G E_{01}}{I G E_{01}}=\frac{-L \int_{0}^{1} S(\widehat{x}) d \widehat{x}}{\left.\widehat{\rho v}\right|_{1} \exp \int_{0}^{1} P_{L} d \widehat{x}^{\prime}-1}
$$

It is clear that when mesh Péclet is high, the exponential dominates and the ratio tends to zero.

Summing up, in the high Péclet regime the ratio $I L E_{01} / I G E_{01}$ tends to 1 ,

and both $I S G E_{01} / I G E_{01}$ and $\widetilde{k}=1 /\left(P_{L 0} I G E_{01}\right)$ become negligible. This asymptotic regime is important as there are extensive areas of a typical flow domain where Péclet is very high. In a practical calculation it is convenient to know the asymptotic values of the coefficients that would otherwise have to be calculated with exponentials of large numbers. Substituting these values in Eqn. 28, the algebraic equation for this high Péclet regime is

$$
(\rho v)_{P} \phi_{P}-(\rho v)_{W} \phi_{W}=\int_{x_{W}}^{x_{P}} S(x) d x
$$

which is consistent with that obtained by integrating the convection equation with source between $W$ and $P$.

\section{Hermite splines and Gauss quadrature}

For the correct evaluation of the discretized coefficients one has to be as accurate as possible in the numerical calculation of the integrals. In one way or another the issue of numerical integration hinges on the interpolation of the function to be integrated by a polynomial of a given degree. Two differ- 
ent approaches have been pursued in this work: Hermite splines and Gauss quadrature integration.

Hermite splines require the values and the derivatives of the function to be interpolated at the edges of the interval. In the formulation presented in this paper these can be evaluated with the exact expressions of the general $\widehat{\phi}$, thus any derivative can be made readily available at the edges. The determination of these derivatives need some factors already calculated in the algebraic nodal equation, so Hermite interpolation is appealing because there is little extra computational effort. To calculate the coefficients that appear in the discretized equations only the integral for a given Hermite spline is required. The interpolating polynomial is a linear combination of the elements of the Hermite basis whose integrals can be calculated beforehand in the domain $(0,1)$ that, due to the mapping, is the same for all intervals.

The simplest Hermite spline is the one that has the same edge values and first derivatives as the function to be interpolated. Its Hermite basis is a set of four polynomials where the first two have the value of one at one edge and zero at the other, with zero first derivative at both. The last two have the same behaviour substituting value for derivative and viceversa. Having four values to be adjusted a third-order polynomial is needed for each element of the basis. The set of four polynomials are referred to as the cubic Hermite spline basis.

When the second derivative at the edges is included the polynomial degree increases two units and logically this also happens when including every new edge derivative. In this work we have employed cubic, quintic and septic Hermite splines. To obtain the coefficients of the algebraic equations only the 
integrals in the unity domain are required that can, in turn, be obtained in terms of the edge values of the function and the derivatives employed. When using Hermite splines as interpolants of a function $f(\widehat{x})$, its integral, written in terms of edge values and derivatives, is

$$
\begin{aligned}
\text { Cubic } \quad \int_{0}^{1} f(x) d x & =\frac{1}{2}(f(0)+f(1))+\frac{1}{12}\left(f^{\prime}(0)-f^{\prime}(1)\right) \\
\text { Quintic } \quad \int_{0}^{1} f(x) d x & =\frac{1}{2}(f(0)+f(1))+\frac{1}{10}\left(f^{\prime}(0)-f^{\prime}(1)\right) \\
& +\frac{1}{120}\left(f^{\prime \prime}(0)+f^{\prime \prime}(1)\right) \\
\text { Septic } \quad \int_{0}^{1} f(x) d x & =\frac{1}{2}(f(0)+f(1))+\frac{3}{28}\left(f^{\prime}(0)-f^{\prime}(1)\right) \\
& +\frac{1}{84}\left(f^{\prime \prime}(0)+f^{\prime \prime}(1)\right)+\frac{1}{1680}\left(f^{\prime \prime \prime}(0)-f^{\prime \prime \prime}(1)\right)
\end{aligned}
$$

For instance, if the integral of the mesh Péclet number is evaluated, and the integrand is approximated with a cubic Hermite spline, its value is

$$
\int_{0}^{1} P_{L}(\widehat{x}) d \widehat{x}=\frac{1}{2}\left(P_{L 0}+P_{L 1}\right)+\frac{1}{12}\left(\left.P_{L}^{\prime}\right|_{0}-\left.P_{L}^{\prime}\right|_{1}\right)
$$

As the approximation of the integrand has to be as accurate as possible we need that the integrands and their derivatives be continuous up to the same degree as the interpolating polynomial. In the case of cubic Hermite the integrand should be continuous up to the third derivative, fifth for quintic and seventh for septic. Note that this is a numerical requirement not a mathematical one, the integrals can still be calculated for a discontinuous integrand. Moreover, if there are discontinuities in the coefficients and/or source we can always place a node in the discontinuity and everything will work fine.

In the evaluation of the integrals by Hermite splines another approach that 
has been considered in this work is to transform first the integrand $1 / \bar{E}$ in the following way

$$
\begin{aligned}
\frac{1}{\bar{E}} & =\exp \int_{\widehat{x}}^{1} P_{L} d \widehat{x}^{\prime}=\exp \int_{\widehat{x}}^{1}\left(P_{L}-\bar{P}_{L}\right) d \widehat{x}^{\prime} \cdot \exp \int_{\widehat{x}}^{1} \bar{P}_{L} d \widehat{x}^{\prime} \\
& =\exp \bar{P}_{L}(1-\widehat{x}) \cdot \exp \int_{\widehat{x}}^{1}\left(P_{L}-\bar{P}_{L}\right) d \widehat{x}^{\prime} \\
& =\exp \bar{P}_{L}(1-\widehat{x}) \cdot \exp \int_{0}^{1}\left(P_{L}-\bar{P}_{L}\right) d \widehat{x}^{\prime} \cdot \exp -\int_{0}^{\widehat{x}}\left(P_{L}-\bar{P}_{L}\right) d \widehat{x}^{\prime}
\end{aligned}
$$

If we choose $\bar{P}_{L}=\int_{0}^{1} P_{L} d \widehat{x}$ the second exponential is one and then

$$
\frac{1}{\bar{E}}=\exp \bar{P}_{L}(1-\widehat{x}) \cdot \exp -\int_{0}^{\widehat{x}}\left(P_{L}-\bar{P}_{L}\right) d \widehat{x}^{\prime}
$$

This strategy continues by approximating the second exponential with Hermite splines. This exponential is much more suitable for being approximated with a polynomial than $\exp \int_{\widehat{x}}^{1} P_{L} d \widehat{x}^{\prime}$ in the case of moderate/large mesh Péclet and in fact this was the rationale behind this transformation. The original exponential can vary a great deal within an interval, its value is $\exp \int_{0}^{1} P_{L} d \widehat{x}^{\prime}$ at the left boundary and 1 at the right boundary. The one after the transformation starts and ends with the same value: 1 . With this approach the $I G E_{01}$ factor for constant $\Gamma$ is

$$
\begin{aligned}
I G E_{01} & =\int_{0}^{1} \frac{1}{\bar{E}} d \widehat{x}=\int_{0}^{1}\left(\exp \bar{P}_{L}(1-\widehat{x}) \cdot \exp -\int_{0}^{\widehat{x}}\left(P_{L}-\bar{P}_{L}\right) d \widehat{x}^{\prime}\right) d \widehat{x} \\
& =\int_{0}^{1} \sum_{j=0}^{n} a_{j} \widehat{x}^{j} \exp \bar{P}_{L}(1-\widehat{x}) d \widehat{x}=\sum_{j=0}^{n} a_{j} \int_{0}^{1} \widehat{x}^{j} \exp \bar{P}_{L}(1-\widehat{x}) d \widehat{x}
\end{aligned}
$$

The integrals in the previous expression can be evaluated exactly because

$$
\int x^{n} e^{b x} d x=\frac{e^{b x}}{b}\left(x^{n}-\frac{n x^{n-1}}{b}+\frac{n(n-1) x^{n-2}}{b^{2}}-\cdots+\frac{(-1)^{n} n !}{b^{n}}\right)
$$


For a n-ic Hermite spline the final result is

$$
\begin{aligned}
I G E_{01} & =\int_{0}^{1} \frac{1}{\bar{E}} d \widehat{x} \\
& =\frac{\exp \bar{P}_{L}-1}{\bar{P}_{L}} \sum_{j=0}^{n} a_{j} \frac{j !}{\bar{P}_{L}^{j}}-\frac{1}{\bar{P}_{L}} \sum_{j=1}^{n} a_{j}\left(1+\sum_{l=1}^{j-1} \frac{j !}{\bar{P}_{L}^{l}(j-l) !}\right)
\end{aligned}
$$

$a_{j}$ being the coefficients of the Hermite spline that interpolates the transformed exponential. If $\Gamma$ varies, that is, $\widehat{\Gamma} \neq 1, a_{j}$ are the coefficients of the Hermite spline that interpolates $1 / \widehat{\Gamma}$ times the transformed exponential.

We found in the cases tested that the interpolation of the original exponential associated to $1 / \bar{E}$ is adequate for mesh Péclet below 5 . The difficulty to approximate the original exponential for higher Péclet may result in the interpolating Hermite spline producing negative integrals for $I G E_{01}$ or $I L E_{01}$ which is not mathematically correct. The coefficients in Eqn. 28 are positive because the integrals contained in them are always positive. So the integral scheme is robust and monotonic as long as the numerical value has the same positive sign. Our computational experience with this scheme is that for mesh Péclet higher than five the new integral for $1 / \bar{E}$ always provided positive results, in contrast to the original one that did not always do so.

We have also checked the use of a 3- and 4-point Gauss-Legendre quadrature for integral evaluation. As all integrals involved are between 0 and 1 the implementation is independent of the problem or the mesh. A n-point Gauss-Legendre quadrature manages to integrate exactly a polynomial of $(2 n-1)$ th degree by calculating the integrand at the Gauss points. As an example, a 3-point quadrature would calculate the integrand at $\widehat{x}_{1}=1 / 2$, $\widehat{x}_{2}=1 / 2(1+\sqrt{3 / 5})$ and $\widehat{x}_{3}=1 / 2(1-\sqrt{3 / 5})$ which are Gauss points for an 
interval $[0,1]$. The generic expression is

$$
\int_{0}^{1} f(\widehat{x}) d \widehat{x}=\frac{1}{2} \sum_{i=1,3} w_{i} f\left(\widehat{x}_{i}\right)
$$

where $w_{i}$ are the weights, namely, $8 / 9$ for the point $1 / 2$ and $5 / 9$ for the remaining two. For example, $\bar{E}$ and $I S$, that are integrals within integrals, are evaluated as

$$
\begin{aligned}
\bar{E}\left(\widehat{x}_{i}\right) & =\exp -\int_{\widehat{x}_{i}}^{1} P_{L} d \widehat{x}^{\prime} \\
& =\exp -\left[\frac{1-\widehat{x}_{i}}{2}\left[\frac{8}{9} P_{L}\left(\widehat{x}_{1}+\widehat{x}_{i} \widehat{x}_{1}\right)+\frac{5}{9}\left(P_{L}\left(\widehat{x}_{2}+\widehat{x}_{i} \widehat{x}_{3}\right)+P_{L}\left(\widehat{x}_{3}+\widehat{x}_{i} \widehat{x}_{2}\right)\right)\right]\right] \\
I S\left(\widehat{x}_{i}\right) & =L \int_{0}^{\widehat{x}_{i}} S d \widehat{x}^{\prime} \\
& =L \frac{\widehat{x}_{i}}{2}\left[\frac{8}{9} S\left(\widehat{x}_{i} \widehat{x}_{1}\right)+\frac{5}{9}\left(S\left(\widehat{x}_{i} \widehat{x}_{2}\right)+S\left(\widehat{x}_{i} \widehat{x}_{3}\right)\right)\right]
\end{aligned}
$$

Similarly, there are additional expressions for 4-point Gauss quadrature. This way of approximating the integrals can never produce negative values as the result is the sum of several positive integrand evaluations. Gauss quadrature provides a robust and monotonic scheme.

Contrary to Hermite splines that only require the value of the function and its derivatives at the nodes, the Gauss-Legendre quadrature needs the values of the integrand at some points within the interval. If the source and the coefficients are prescribed analytically it is straightforward to obtain them, but in a general case where $v$ is obtained via its transport equation and/or the source is not analytical, it may be a bit cumbersome to get the values at the Gauss points. In this respect Hermite splines are very appealing because there is no need of information inside the interval. 


\subsection{Estimated rate of convergence of Hermite splines}

In this section an assessment of the errors involved in the evaluation of the integrals and the coefficients of the discretized equation is carried out. We will start by describing the errors in approximating the integrand, finishing with the spatial discretization error, that is, the difference between the exact and the numerical solution.

Let $H_{N}^{(k)}(f ; \widehat{x})$ be the Hermite polynomial of degree $N$ that approximates a function $f$ in the interval $(0,1)$. The interpolating polynomial is such that it has the same values as the function and its derivatives up to order $k$ at 0 and $1(N=1+2 k)$. Then (see for instance [25]),

$$
f(\widehat{x})-H_{N}^{(k)}(f ; \widehat{x})=\frac{f^{N+1}(t)}{(N+1) !} \widehat{x}^{k+1}(\widehat{x}-1)^{k+1} \quad ; \quad 0<t<1
$$

and

$$
\int_{0}^{1}\left|f(\widehat{x})-H_{N}^{(k)}(f ; \widehat{x})\right| d \widehat{x} \leq \max _{0<t<1}\left|f^{N+1}(t)\right| \frac{1}{(N+1) !} \int_{0}^{1} \widehat{x}^{k+1}(1-\widehat{x})^{k+1} d \widehat{x}
$$

$f^{N+1}$ is the $(\mathrm{N}+1)$ th derivative w.r.t $\widehat{x}$ of the function to be interpolated. The integral can even be written in terms of $k$

$$
\int_{0}^{1} \widehat{x}^{k+1}(1-\widehat{x})^{k+1} d \widehat{x}=\frac{(k+1) !}{2^{k+1}} \frac{1}{1 \cdot 3 \cdot 5 \cdots(2 k+3)}
$$

and finally

$$
\int_{0}^{1}\left|f(\widehat{x})-H_{N}^{(k)}(f ; \widehat{x})\right| d \widehat{x} \leq C(N) \max _{0<t<1}\left|f^{N+1}(t)\right|=C(N) l^{N+1} \max _{x_{l b}<\xi<x_{r b}}\left|f^{N+1}(\xi)\right|
$$


because $d x=l d \widehat{x}, l$ being the interval length. $C(N)$ is a constant that depends on the polynomial degree. Thus, the error in the coefficients is formally of order $N+1$. Cubic Hermite is fourth-order $(\mathrm{k}=1, \mathrm{~N}=3)$, quintic Hermite is sixth-order $(k=2, N=5)$ and septic Hermite is eighth-order $(k=3, N=7)$.

Let $L^{N}$ and $f^{N}$ be the numerical matrix of coefficients and the numerical source respectively and let $L$ and $f$ be the exact ones. In order to estimate the spatial discretization error $\left\|u^{N}-u\right\|$ note that

$$
L^{N}\left(u^{N}-u\right)=f^{N}-L^{N} u=f^{N}-f-\left(L^{N}-L\right) u
$$

and then

$$
\left\|u^{N}-u\right\| \leq \frac{\left\|f^{N}-f\right\|}{\left\|L^{N}\right\|}+\frac{\left\|L-L^{N}\right\|}{\left\|L^{N}\right\|}\|u\|
$$

We have followed a standard notation, so now $L$ is a linear operator and $l$ will be the interval size. The first term is the error in the source and the second is the error in the coefficients, both contribute in the same manner to the discretization error. As the numerators are formally of order $N+1$ the whole discretization error is of order $N+1$ if the denominator is independent of $l$. The inequality is satisfied by any norm but the easiest one to estimate is the infinity norm. $\left\|L^{N}\right\|_{\infty}$ is of order $\rho v$ for high Péclet and of order $\Gamma / l$ for low Péclet. For high Péclet $\left\|L^{N}\right\|_{\infty}$ does not depend on $l$, for low Péclet $\left\|L-L^{N}\right\|_{\infty}$ is of order $l^{N}$ but the spatial discretization error is still of order $l^{N+1}$. 


\section{Results}

The ENATE approach was applied to three different test cases, two linear and one nonlinear. In all cases three norms of the residuals were evaluated $L_{1}, L_{2}$ and $L_{\infty}$, calculated as

$$
\begin{aligned}
L_{1} & =\frac{1}{n-1} \sum_{i=1}^{n-1}\left|\phi_{\text {calc }}\left(x_{i}\right)-\phi_{\text {exact }}\left(x_{i}\right)\right| \\
L_{2} & =\sqrt{\frac{1}{n-1} \sum_{i=1}^{n-1}\left(\phi_{\text {calc }}\left(x_{i}\right)-\phi_{\text {exact }}\left(x_{i}\right)\right)^{2}} \\
L_{\infty} & =\max _{i}\left|\phi_{\text {calc }}\left(x_{i}\right)-\phi_{\text {exact }}\left(x_{i}\right)\right|
\end{aligned}
$$

being $n-1$ the number of internal nodes. In the second case where the value at the right end of the domain is also calculated, the number of points used in the norms is $n$. In all cases a uniform mesh has been employed.

In order to compare our scheme with more traditional ones employed in the solution of second-order ODEs, we have also calculated the three cases with the central-differencing scheme (CDS) and a compact scheme (CS) recently proposed [23]. As explained by Sen, this compact scheme has a three-point stencil and is formally fourth-order. It solves for the variable and its first derivative in a compact way. Due to its three-node stencil we could use the same algorithm (Tridiagonal Matrix Algorithm, TDMA) to solve the system of equations. As will be seen the rates of convergence of both behave according to theory in all cases tested. From our point of view it was more interesting to assess if for a given number of nodes their residuals were lower or higher than those produced by our numerical integration. 


\subsection{Case with $\Gamma$ variable}

The first test case is one employed by Tian and Dai [24] to assess the accuracy of finite volume schemes of spectral resolution. In this case the velocity is constant and the diffusion coefficient varies linearly with the position. The transport equation is

$$
\frac{d}{d x}\left(\rho v \phi-\Gamma \frac{d \phi}{d x}\right)=S(x) \quad ; \quad v(x)=1+\epsilon \quad ; \quad \Gamma(x)=\epsilon(1+x)
$$

The source is $S(x)=e^{x}(1-\epsilon(1+x))$. The factor $\epsilon$ controls the value of the Péclet number of the whole domain, which can vary from a value of order one to infinity. With boundary conditions $\phi(0)=1+1 / 2^{1 / \epsilon}$ and $\phi(1)=e+2$, the solution is given by

$$
\phi(x)=e^{x}+(1+x)\left(\frac{1+x}{2}\right)^{1 / \epsilon}
$$

Although the source is very smooth the solution develops a boundary layer near $x=1$ for small $\epsilon$ with thickness of the order of $\epsilon$. For infinite Péclet number $(\epsilon=0)$ the solution is $e^{x}$ for the whole domain with a discontinuity of value 2 at $x=1$. The solution for $\epsilon=10^{-2}$ is shown in Fig. 1.

As the problem has an analytical solution and Eqn. 28 provides the exact solution, all factors in the discretized equation should also be analytical functions of $x$. In fact, by performing all integrations it can be found that the discretized equation following the ENATE procedure is

$$
\begin{aligned}
\left(\rho v \widetilde{k}_{W P}+\rho v\left(1+\widetilde{k}_{P E}\right)\right) \phi_{P} & =\rho v\left(1+\widetilde{k}_{W P}\right) \phi_{W}+\rho v \widetilde{k}_{P E} \phi_{E} \\
& +\rho v\left(e^{x_{P}}-e^{x_{E}}\right) \widetilde{k}_{P E} \\
& -\rho v\left(e^{x_{W}}-e^{x_{P}}\right)\left(1+\widetilde{k}_{W P}\right)
\end{aligned}
$$




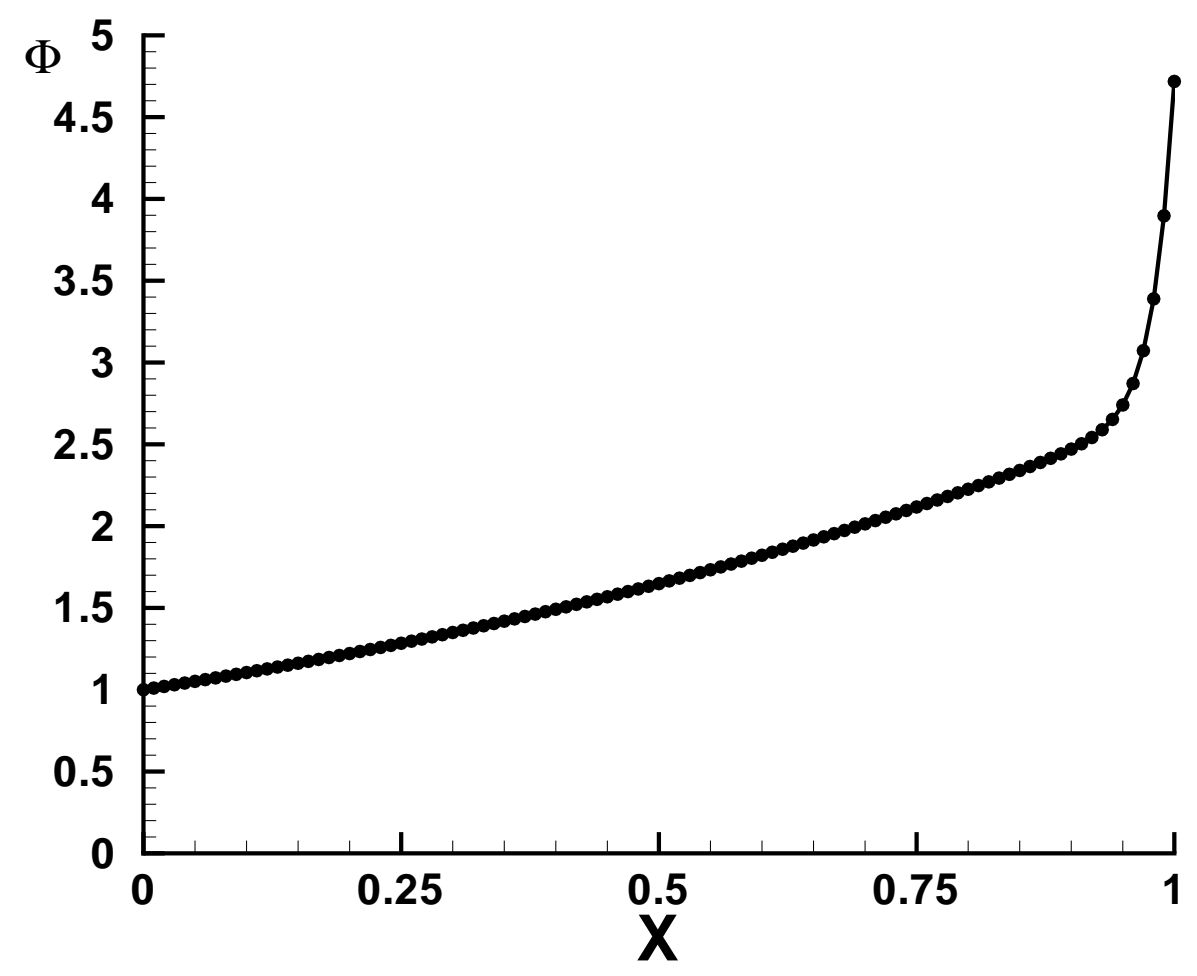

Fig. 1. Solid line: Exact Solution for $\epsilon=10^{-2}$. Black dots: Computed solution with 100 nodes

$$
\text { where } \quad \widetilde{k}_{i i+1}=\left[\left(\frac{1+x_{i+1}}{1+x_{i}}\right)^{(1+\epsilon) / \epsilon}-1\right]^{-1}
$$

and that this algebraic equation provides the exact solution. The variable $\phi^{\text {new }}=\phi-e^{x}$ is the one governed by the source-free algebraic equation

$$
\left(\rho v \widetilde{k}_{W P}+\rho v\left(1+\widetilde{k}_{P E}\right)\right) \phi_{P}^{n e w}=\rho v\left(1+\widetilde{k}_{W P}\right) \phi_{W}^{n e w}+\rho v \widetilde{k}_{P E} \phi_{E}^{n e w}
$$

Remember that in the theoretical derivation $\bar{\phi}^{N}$ represented the variable with no source, so in every interval there must be a connection between $\bar{\phi}^{N}$ and $\phi^{\text {new }}$, i.e., $\bar{\phi}^{N}=c_{1} \phi^{\text {new }}+c_{2}$, and then $F=c_{1} e^{x}+c_{3} . c_{1}, c_{2}$ and $c_{3}$ are constants included to satisfy the boundary conditions. This comment on the connections between these variables is just to convey the idea that for an arbitrary nonho- 
mogeneous convection diffusion equation that possesses an analytic solution one should be able to spot the variables $F$ and $\bar{\phi}^{N}$ that conform its solution just by calculating all integrals exactly and deriving the algebraic equation above.

For a general problem the integrands do not have a primitive function so it is important to assess the accuracy of a numerical integration procedure in all integrals involved. In this case $\widehat{\rho v}=1$ so $I L E_{01}=I G E_{01} . I G E_{01}$ appears in many factors of the algebraic equation and was calculated through the polynomial interpolation of the integrand, that is, it was not calculated with the exact expression $I G E_{01}=I L E_{01}=\left(\exp \int_{0}^{1} P_{L} d \widehat{x}-1\right) / P_{L 0}$. In doing so, the accuracy of the polynomial integration was evaluated. Later, we found that the difference between the exact expression and the polynomial interpolation was negligible. For instance, the $L_{\infty}$ norm of the difference between the two ways of calculating $I G E_{01}$ with 101 nodes is of the order of $10^{-10}$ per unit of $I G E_{01}$.

For this case the $L_{2}$ norms of the residuals are plotted in Fig. 2 for different interval sizes of evenly distributed nodes. The interval sizes range from $\Delta x=10^{-1}$ to $\Delta x=10^{-4}$. The other norms are not plotted because they behave in the same way as the $L_{2}$ norm for all cases. The factor $\epsilon$ is $10^{-2}$. As can be seen in the figure septic Hermite interpolation produces a seventhorder scheme for $\Delta x$ around $10^{-1}$ that converts to a eight-order scheme for $\Delta x$ less than $5.010^{-2}$. Quintic Hermite has the same behaviour in those intervals: fifth-order and sixth-order convergence. As cubic Hermite is fourth-order over a wide range of $\Delta x$ the rate of convergence of the Hermite splines is in accordance with the theory described earlier. For septic Hermite the $L_{2}$ norm is $10^{-7}$ with 9 internal nodes so it can reach machine accuracy already for 


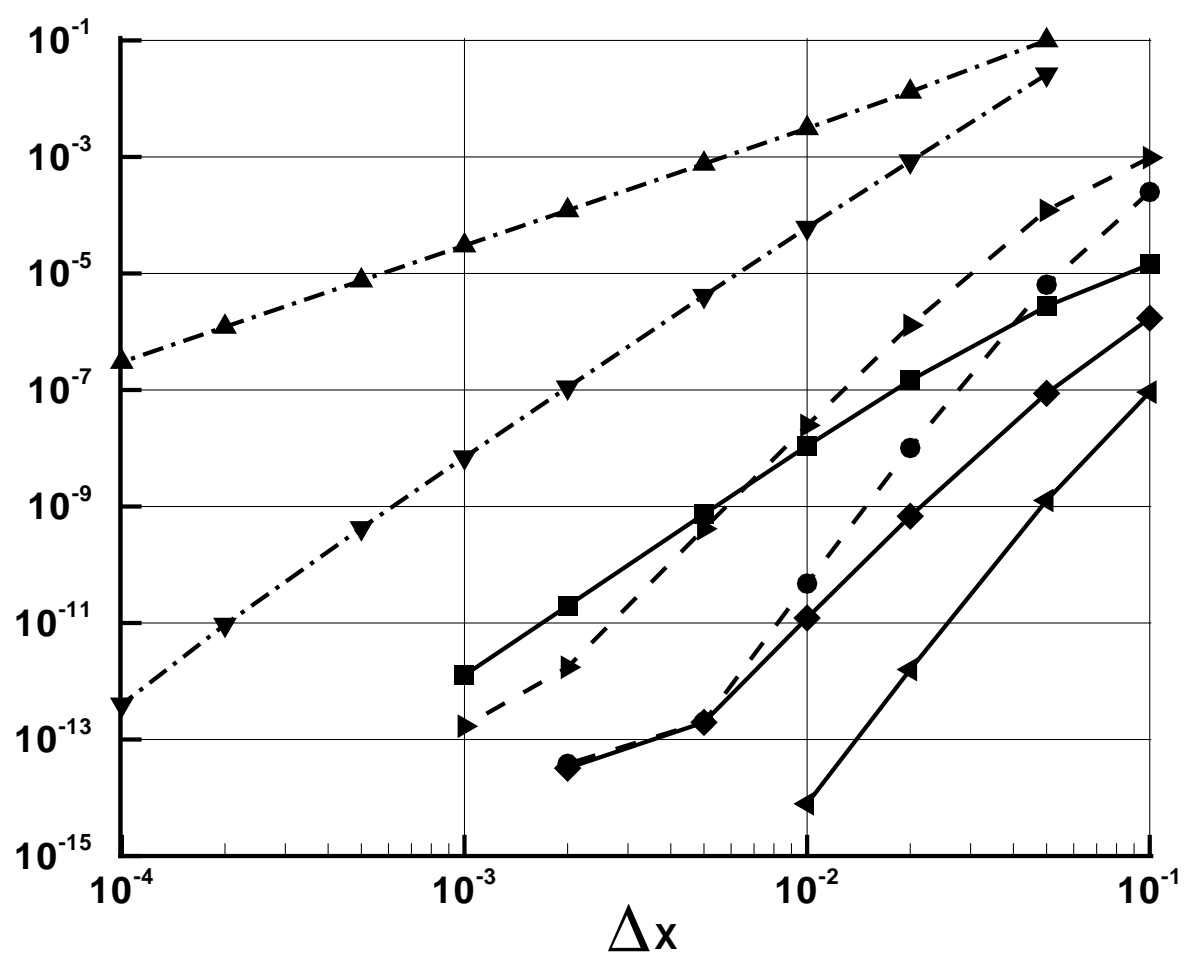

Fig. 2. Energy norm of the residuals for $\epsilon=10^{-2}$. Dashed lines correspond to Gauss quadrature, solid lines to Hermite splines and dash-dotted lines to finite-volume or finite-difference schemes explained in the text $>$-point Gauss - 4-point Gauss Cubic Hermite $\downarrow$ Quintic Hermite \ Septic Hermite Central differencing $\mathbf{\nabla}$ Compact scheme

101 nodes (99 internal nodes, $\Delta x=10^{-2}$ ). All Gauss quadrature evaluations provide higher errors than those obtained with Hermite splines of the modified exponential, although the rates of convergence of 3-point and 4-point Gauss are similar to those of quintic and septic Hermite. This seems logical because 3-point and 4-point Gauss quadrature procedures are able to integrate exactly a polynomial of fith- and seventh-degree, the same degrees as the quintic and septic Hermite polynomials. The residuals for CDS and CS are also plotted, the former is second-order and the latter is fourth-order as shown in the figure. The difference between the errors of the schemes with integral evaluation and 
those that evaluate the derivatives of the original ODE is notable. As an extreme example, septic Hermite with fifty nodes has almost the same accuracy as the compact scheme with ten thousand.

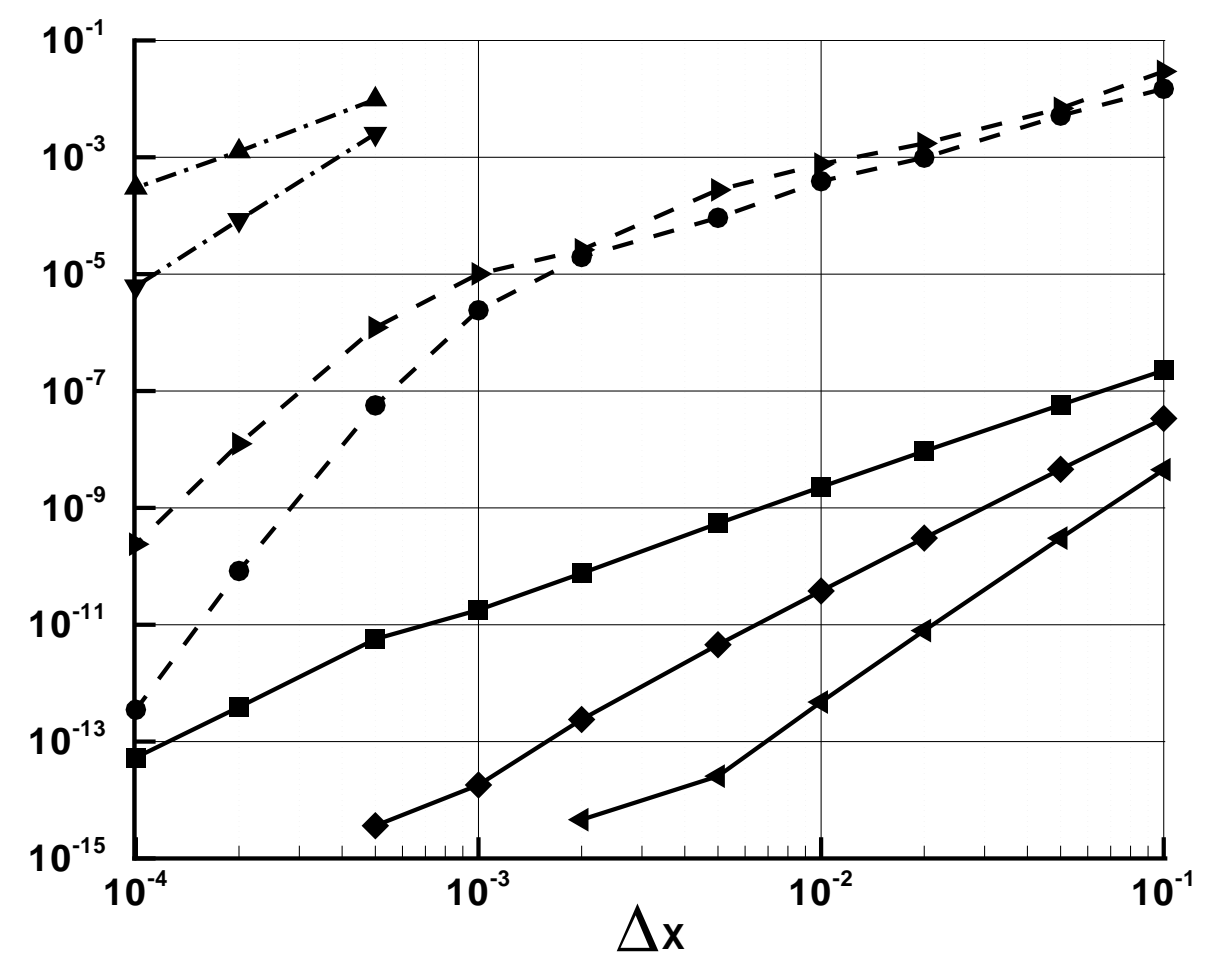

Fig. 3. Energy norm of the residuals for $\epsilon=10^{-4}$. Same symbols as Fig. 2

The rates of convergence depend on the Péclet number of the whole domain through the parameter $\epsilon$. This issue is depicted in Fig. 3 where the same norms are shown for $\epsilon=10^{-4}$. Septic Hermite is almost reaching machine accuracy for 201 nodes. The behaviour of 3- and 4-point Gauss quadrature contains two regions. In the first region both are almost second-order but from $\mathrm{N}=1001$ until $\mathrm{N}=10001$ three-point Gauss is fourth-order and four-point Gauss is seventhorder. This may be related to the accuracy of the exponential approximation. Gauss quadrature provides the exact integral if the integrand is a polynomial of a given degree but, as it is an exponential what is being approximated 
the errors may be significant for large $\Delta x$. Although the rate of convergence is high for $\Delta x$ small, the fact that they start with very large residuals with few nodes, and that across two decades the reduction is only four orders of magnitude, makes them require many more nodes to reach machine accuracy than Hermite splines. Apart from the accuracy of the coefficients, Hermite splines can handle very large Péclet numbers because $I G E_{01}$ and $I S G E_{01}$ are calculated with Eqn. 49, the only difference being the $a_{j}$ coefficients. In the large Péclet region the first exponential dominates so the ratio $I S G E_{01} / I G E_{01}$ is

$$
\frac{I S G E_{01}}{I G E_{01}}=\frac{\left.\sum_{j=0}^{n} a_{j} \frac{j !}{\bar{P}_{L}^{j}}\right|_{I S G E}}{\left.\sum_{j=0}^{n} a_{j} \frac{j !}{\bar{P}_{L}^{j}}\right|_{I G E}}
$$

expression that does not contain any large exponential.

For this case, septic Hermite behaves like a 4th order scheme. A striking result is that with 11 nodes the $L_{2}$ norm of the error is between one and two orders of magnitude less than that in Fig 2. The reason is that mentioned above, for large mesh Péclet the exponential term dominates and the errors made in the $a_{j}$ are only felt in the first factor of Eqn. 49, whereas if the second factor is not negligible the errors in the $a_{j}$ affect more strongly the solution. The order of convergence of CDS and CS is that expected although the region where both CDS and CS are stable is very limited. It can be theoretically shown that the stable region for $\mathrm{CDS}$ is $\mathrm{Pe}<2$ and for $\mathrm{CS}$ is $\mathrm{Pe}<8 / 3$, based on the interval size. 


\subsection{Case with $v$ variable}

The second test case was obtained from ten Thije Boonkamp and Anthonissen [21]. The solved transport equation is

$$
\begin{aligned}
& \frac{d}{d x}\left(\rho v \phi-\Gamma \frac{d \phi}{d x}\right)=S(x) ; \quad v(x)=(1+x)^{3} \quad ; \quad \Gamma / \rho=\epsilon=\mathrm{const} \\
& S(x)=\frac{S_{\max }}{1+S_{\max }(2 x-1)^{2}}
\end{aligned}
$$

The boundary conditions are $\phi(0)=0$ and $\phi^{\prime}(1)=0$. The velocity field does not correspond to a real fluid because $\rho v$ is not constant, but as in several dimensions the velocity field of a real fluid can vary along one coordinate, it is interesting to check the behaviour of the ENATE approach when the convective flux is not constant. If $S_{\max } \gg 1$ the source starts and finishes with a value of order one at the edges of the domain and it goes up to $S_{\max }$ at $x=1 / 2$. $S_{\max }$ can be changed to adjust the thickness and intensity of a steep layer in the solution near $x=0.5$. This case does not have an analytic solution but to realize the effect of $S_{\text {max }}$, a computed solution for $\epsilon=10^{-2}$ with 100 nodes is shown in Fig. 4 for two values of $S_{\max }, 10^{2}$ and $10^{3}$. The steeper gradient near $x=0.5$ can be appreciated as $S_{\max }$ is increased.

The interest of this case is twofold: first, it contains a variable velocity that changes an order of magnitude across the unity domain and second, it possesses a source whose intensity and gradients are adjustable via a parameter $S_{\max }$. An additional reason for choosing this example is to show how boundary conditions of von Newmann type can be handled following this approach. At the end of the last interval the general expression for the derivative is

$$
\left.\frac{1}{P_{L 1}} \frac{d \phi}{d \widehat{x}}\right|_{\widehat{x}=1}=\left[\left.\frac{1}{P_{L 1}} \frac{d F}{d \widehat{x}}\right|_{\widehat{x}=1}+\left.\frac{1}{P_{L 1}}(1-F(1)) \frac{d \bar{\phi}^{N}}{d \widehat{x}}\right|_{\widehat{x}=1}\right]\left(\phi_{N}-\phi_{N-1}\right)(64)
$$




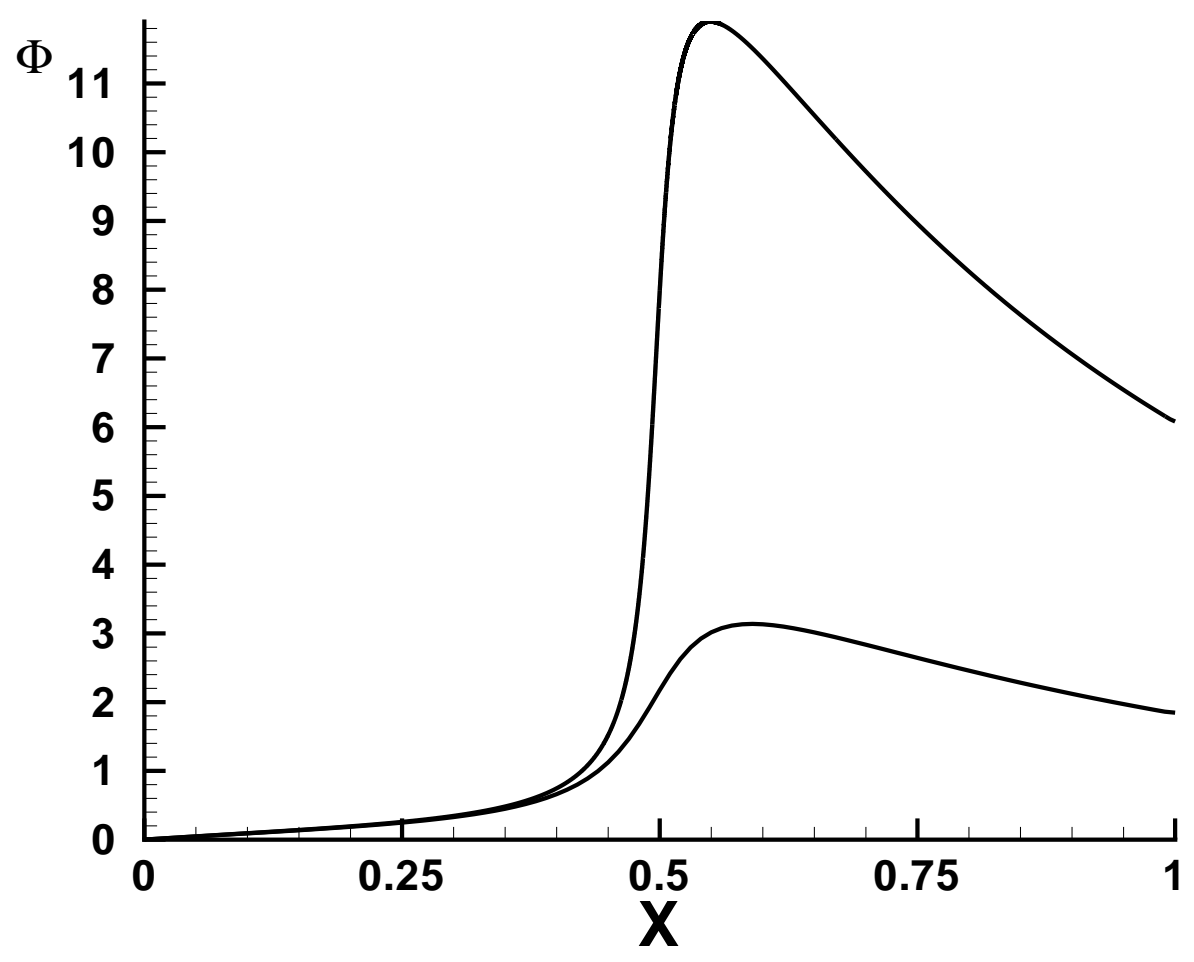

Fig. 4. Computed solution with 101 nodes for two values of $S_{\max }, 10^{2}$ and $10^{3}$. and this derivative is zero due to the boundary condition. Substituting the values of the derivatives the expression that links $\phi_{N}$ (boundary) and $\phi_{N-1}$ (last internal node) is

$$
\phi_{N}\left(1+\frac{\widetilde{k}}{\widehat{\rho v}(1)}\right)=\frac{\phi_{N-1}}{\widehat{\rho v}(1)}\left(\widetilde{k}+\frac{I L E_{01}}{I G E_{01}}\right)+\frac{1}{\left.\rho v\right|_{N}}\left[I S_{01}-\frac{I S G E_{01}}{I G E_{01}}\right]
$$

In the expression above all factors are those of the last interval. The first iteration starts with $\phi_{N}=\phi_{N-1}$ and in subsequent iterations the value at the boundary is updated according to Eqn. 65.

In Fig. 5 and 6 results obtained for $S_{\max }=10^{2}, 10^{3}$ with $\epsilon=10^{-2}$ are plotted. As there is no analytic solution to compare with, the solution with septic Hermite and 10001 nodes was assumed as the reference solution. As seen in 


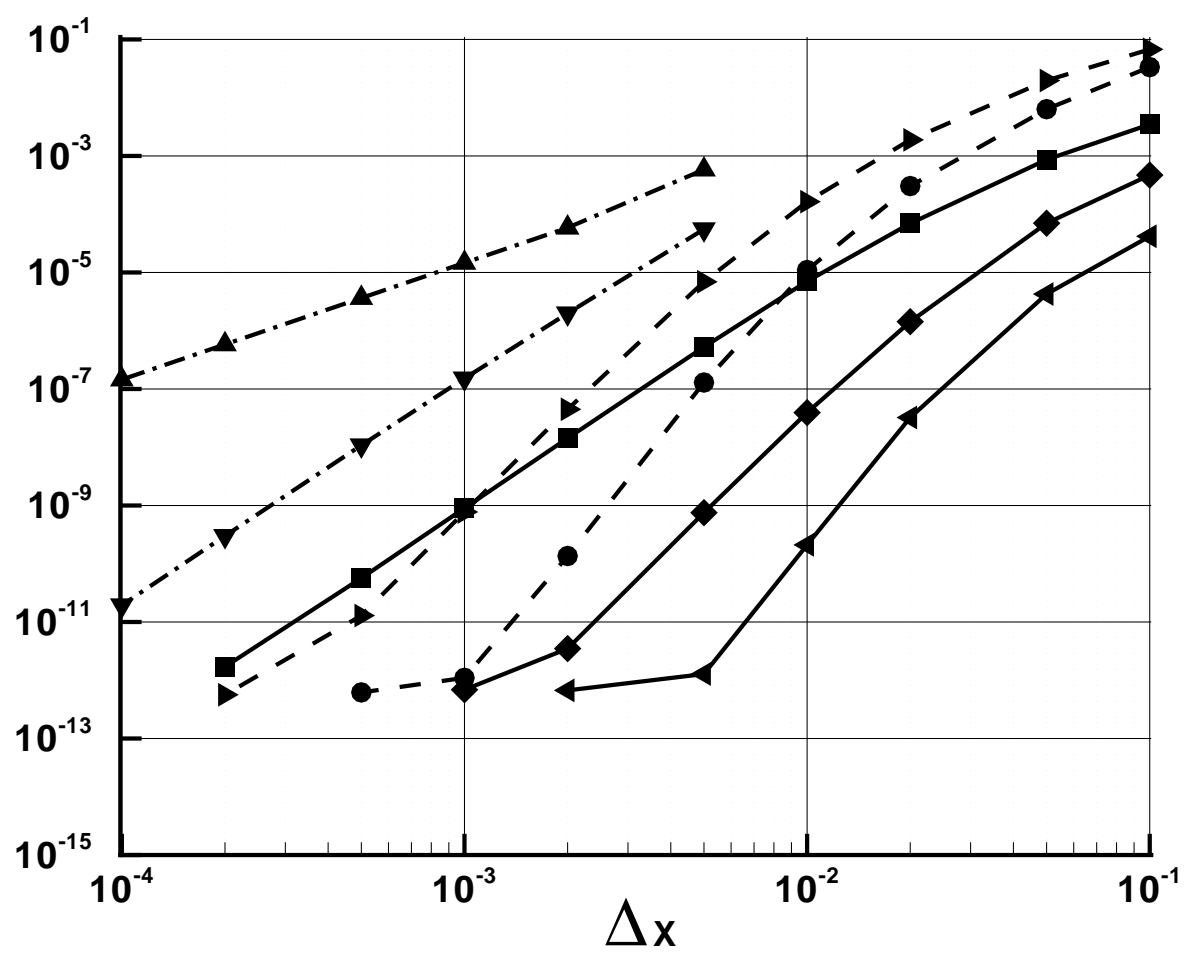

Fig. 5. Residuals for $\epsilon=10^{-2}$ and $S_{\max }=100$. Dashed lines correspond to Gauss quadrature, solid lines to Hermite splines and dash-dotted lines to finite-volume or finite-difference schemes explained in the text $\$$-point Gauss - 4-point Gauss $\square$ Cubic Hermite Quintic Hermite $\triangleleft$ Septic Hermite $\boldsymbol{\Delta}$ Central differencing $\mathbf{\nabla}$ Compact scheme

the figure all schemes have an error of the order of $10^{-12}$ or less in the limit of small $\Delta x$, so the asymptotic solution of all schemes is the same up to the 11th decimal place at least.

For $S_{\max }=10^{2}$ quintic and septic Hermite perform better than a Gauss quadrature strategy over the whole range of interval sizes. From $\Delta x=10^{-2}$ downwards the rate of convergence of all Hermite splines is in accordance with theory until $10^{-12}$ where round-off errors seem to come into play and the rate of convergence is significantly reduced. The residual associated to 
the asymptotic regime of very high mesh Péclet numbers for any integral scheme is $2.0110^{-2}$ (not drawn). This was calculated with $I G E_{01}=I L E_{01}$ and $I S G E_{01} / I G E_{01}$ and $\widetilde{k}$ zero. For relatively large $\Delta x$ (high mesh Péclet) this solution is better than that with Gauss quadrature and it seems to suggest that large exponentials are not very well resolved by Gauss quadrature and that $I G E_{01} / I L E_{01}$ in the algebraic equation should be closer to one than the value calculated with the quadrature.

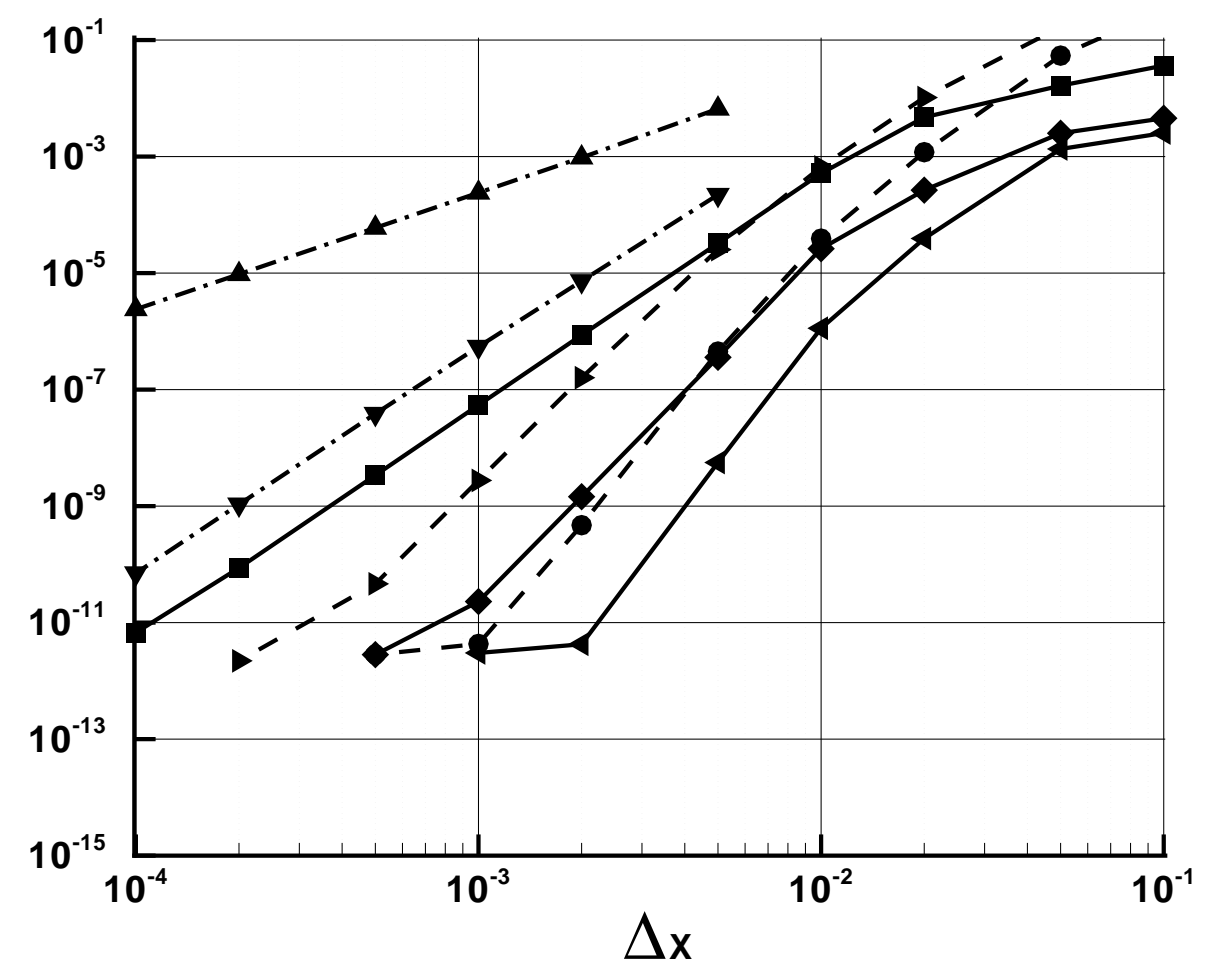

Fig. 6. Residuals for $\epsilon=10^{-2}$ and $S_{\max }=1000$. Same symbols as Fig. 5

Although the actual residual values are different the whole picture is very much the same for $S_{\max }=10^{3}$. There is also a wide region where the integral schemes behave according to theory, especially cubic Hermite. For $S_{\max }=10^{3}$ the residual of very high mesh Péclet for any integral scheme is now of order $10^{-1}$, again better than Gauss quadrature for $\Delta x$ above $5.010^{-2}$, which gives 
more credit to the claim that Gauss quadrature is not able to handle large exponentials in the integrands.

The same results are plotted in the next two figures, 7 and 8 , for $\epsilon=10^{-4}$. As happened in the first test case the residuals for large $\Delta x$ are two orders of magnitude better than for $\epsilon=10^{-2}$ but the rate of convergence is significantly lower, yet it is independent of the source intensity. For both $S_{\max }$ septic Hermite spline behaves like a scheme of roughly fourth order, cubic is second order and quintic third order. There are two distinct regions in the convergence of 3and 4-point Gauss quadrature. In the first one, until $\Delta x=10^{-3}$, they behave like a first-order scheme but from then on their slopes are close to quintic and septic Hermite. The norms for the asymptotic high Péclet regime are $2.010^{-4}$ and $10^{-3}$, better than Gauss quadrature for a wide range of large $\Delta x$. For $\epsilon=10^{-4}$ there is no stable solution with CDS or CS. The one depicted in the figure corresponds to upwind differencing, a first-order scheme.

\subsection{Nonlinear convection-diffusion equation}

The nonlinear convection diffusion equation employed as the last test case is given by

$$
v \frac{d v}{d x}=\mu \frac{d^{2} v}{d x^{2}} \quad \Rightarrow \quad \frac{d}{d x}\left(\frac{1}{2} v^{2}-\mu \frac{d v}{d x}\right)=0
$$

in a unity domain. The prescribed boundary conditions are $v(0)=1$ and $v(1)=0$. This case has an analytic solution given by

$$
v(x)=C_{1} \tanh \left(\frac{C_{1}}{2 \mu}(1-x)\right) \quad \text { with } \quad 1=C_{1} \tanh \left(\frac{C_{1}}{2 \mu}\right)
$$




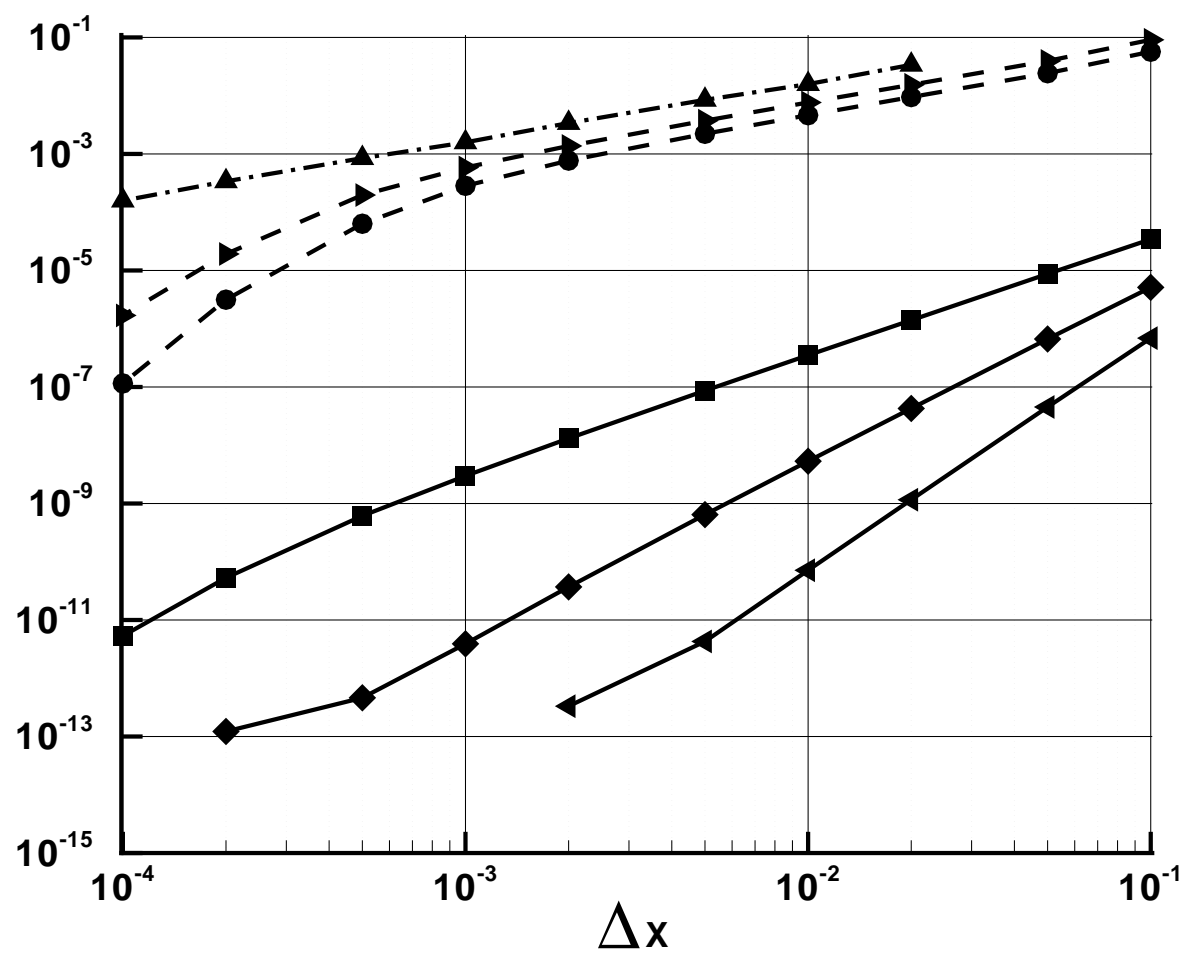

Fig. 7. Residuals for $\epsilon=10^{-4}$ and $S_{\max }=100$. Dashed lines correspond to Gauss quadrature, solid lines to Hermite splines and dash-dotted lines to finite-volume or finite-difference schemes explained in the text $\$$-point Gauss - 4-point Gauss Cubic Hermite Quintic Hermite $\triangleleft$ Septic Hermite $\boldsymbol{\Delta}$ Upwind differencing

In Fig. 9 the solutions for $\mu=0.04$ and $\mu=0.01$ are depicted for the second half of the domain. In the first half the solution is constant and equal to one. It is observed the development of the boundary layer near $x=1$ as $\mu$ is decreased.

If we used Gauss quadrature to calculate the integrals associated to $\bar{E}$ we would require the values of $v$ at intermediate points within the intervals. In the other two cases these were easily obtained, but in this nonlinear case $v$ is the updated variable of the iterative process. The values of $v$ could be 


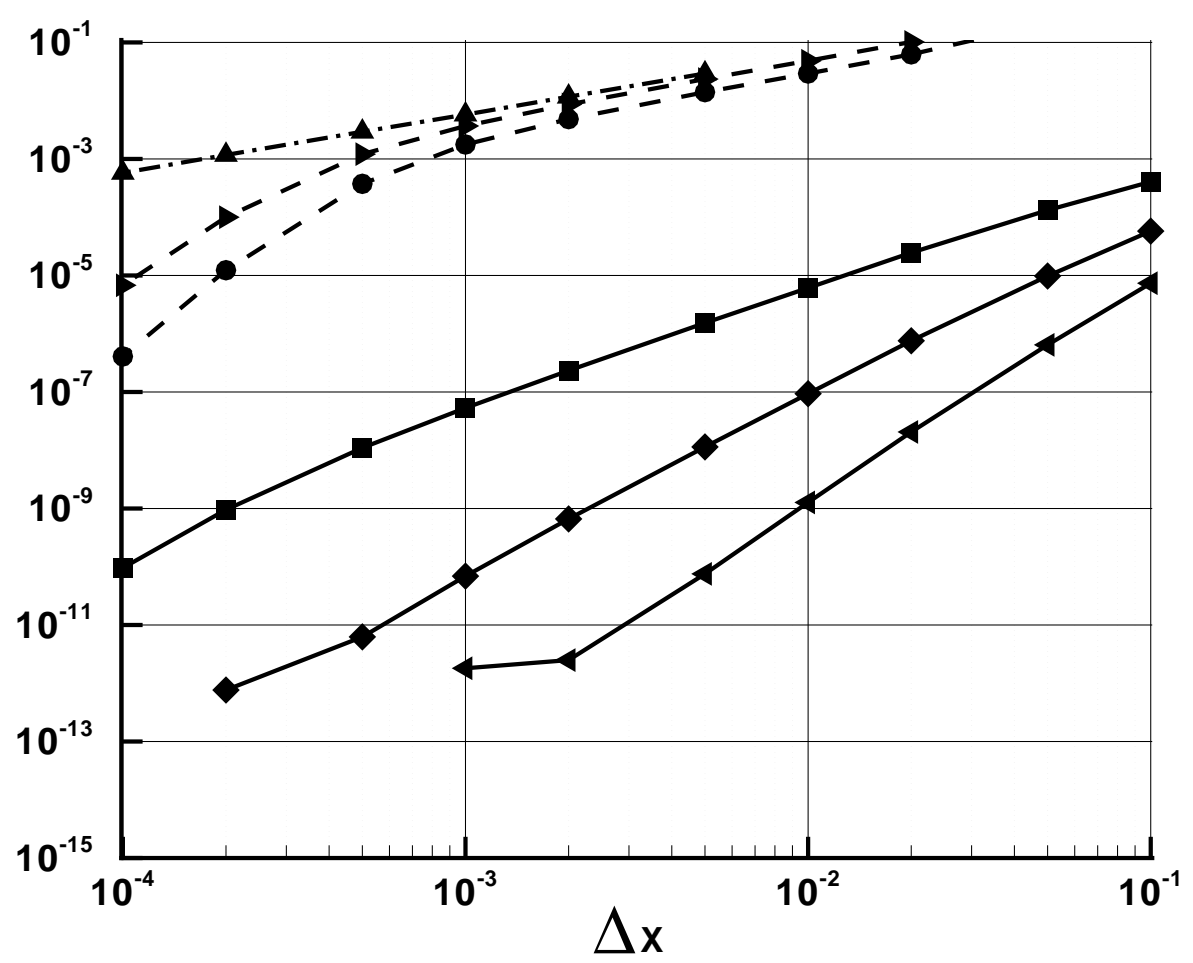

Fig. 8. Residuals for $\epsilon=10^{-4}$ and $S_{\max }=1000$. Same symbols as Fig. 7 obtained based on the exact solution at every iteration but this, in turn, contains the evaluation of integrals that again need the values at intermediate points of the subinterval within the interval, and so on. As there is no way of circumventing this problem we have only employed Hermite splines for the nonlinear equation.

As with the first test case the integrals associated to the coefficients have an analytical primitive. For instance, the integrating factor $E(\widehat{x})=\exp \int^{\widehat{x}} P_{L} d \widehat{x}^{\prime}$ is $(\cosh f(\widehat{x}))^{-1}, f(\widehat{x})$ being $C_{1}\left(1-x_{l b}-l \widehat{x}\right) /(2 \mu)$. In this equation it was also checked that the exact solution could be obtained if the coefficients given in Eqn 28 were analytically calculated. There is a difference with the first test because being a nonlinear equation the coefficients in this equation depend on the solution, whereas those of the first test may be calculated with the spatial 


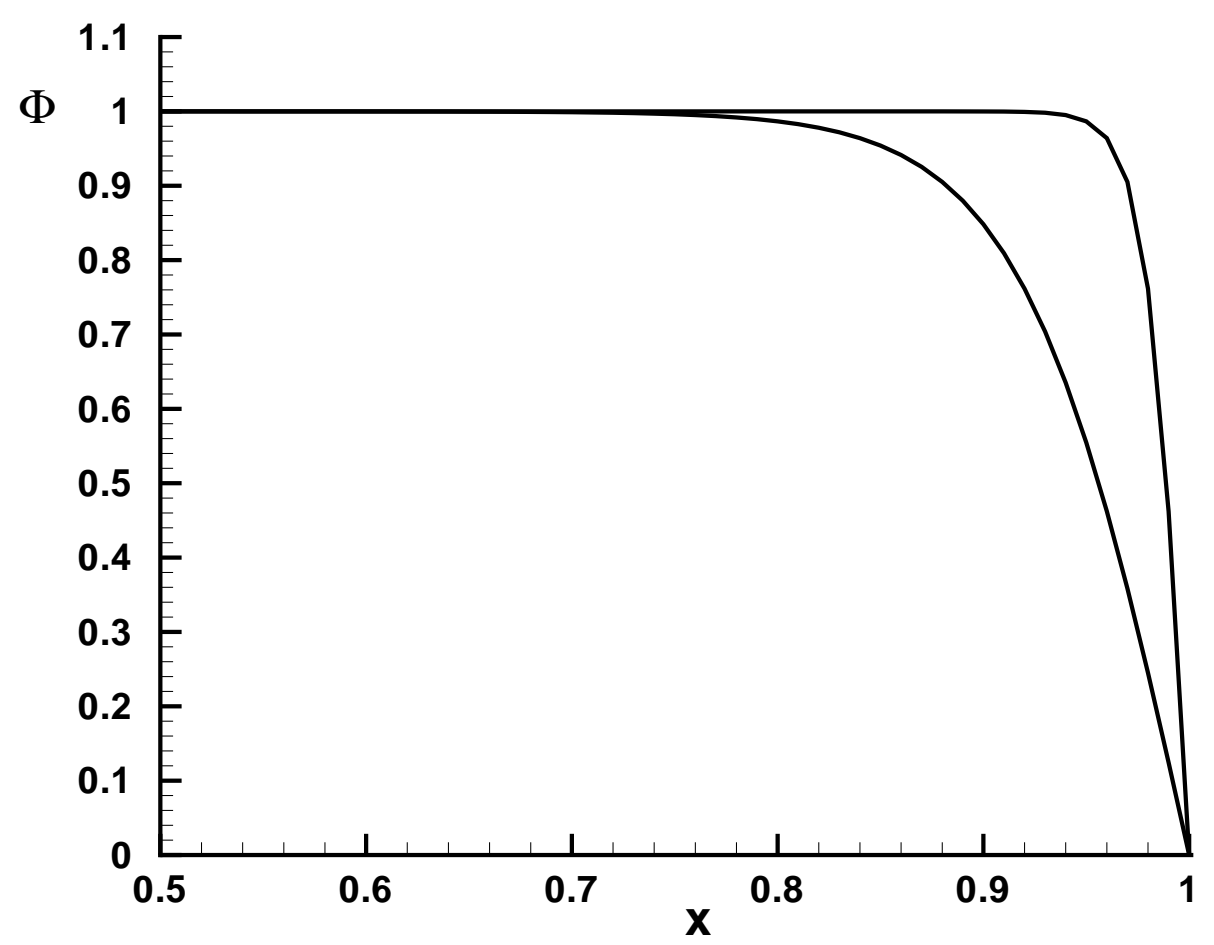

Fig. 9. Solutions for $\mu=0.04$ and $\mu=0.01$

dependence of the convective flux and diffusion coefficients.

In the Appendix the velocity derivatives are derived, which are then used to obtain the mesh Péclet number derivatives of a generic variable $\phi$. These are required to obtain their values at the edges of the interval. In the case of this equation where $\phi=v$ these derivatives are much simpler as there is no source and $\mu=\Gamma$ is constant. The superscript $v$ will be omitted as there is no distinction between $\phi$ and $v$.

$$
\begin{aligned}
& \frac{d \widehat{v}}{d \widehat{x}}=P_{L} \frac{v}{\Delta v}+\frac{1-P_{L 0} \frac{v_{l b}}{\Delta v} I L E_{01}}{I G E_{01}} \\
& \frac{d P_{L}}{d \widehat{x}}=P_{L}^{2}+\frac{P_{L}^{(\Delta v)}-P_{L 0}^{2} I L E_{01}}{I G E_{01}}=P_{L}^{2}+C_{1} \\
& \frac{d^{2} \widehat{v}}{d \widehat{x}^{2}}=\frac{1}{\Delta v} \frac{d}{d \widehat{x}}\left(P_{L} v\right)=\frac{1}{P_{L}^{(\Delta v)}} \frac{d}{d \widehat{x}} P_{L}^{2}
\end{aligned}
$$




$$
\frac{d^{2} P_{L}}{d \widehat{x}^{2}}=\frac{d}{d \widehat{x}} P_{L}^{2} \Rightarrow \frac{d^{3} P_{L}}{d \widehat{x}^{3}}=2\left[\left(\frac{d P_{L}}{d \widehat{x}}\right)^{2}+P_{L} \frac{d^{2} P_{L}}{d \widehat{x}^{2}}\right]
$$

In the second expression note that $C_{1}$ is a constant. If Hermite splines are used for the interpolation, $I L E_{01}$ and $I G E_{01}$ require the derivatives and the values of their integrands. For instance,

$$
I L E_{01}=\int_{0}^{1} \frac{\hat{\lambda}}{\bar{E}} d \widehat{x}^{\prime}=\frac{1}{P_{L 0}} \int_{0}^{1} \frac{P_{L}}{\bar{E}} d \widehat{x}^{\prime}
$$

and

$$
\begin{aligned}
& \frac{d}{d \widehat{x}}\left(\frac{P_{L}}{\bar{E}}\right)=P_{L} \frac{-P_{L}}{\bar{E}}+\frac{1}{\bar{E}} \frac{d P_{L}}{d \widehat{x}}=\frac{1}{\bar{E}}\left(\frac{d P_{L}}{d \widehat{x}}-P_{L}^{2}\right)=\frac{C_{1}}{\bar{E}} \\
& \frac{d^{2}}{d \widehat{x}^{2}}\left(\frac{P_{L}}{\bar{E}}\right)=\frac{d}{d \widehat{x}}\left(\frac{C_{1}}{\bar{E}}\right)=-C_{1} \frac{P_{L}}{\bar{E}} \Rightarrow \frac{d^{3}}{d \widehat{x}^{3}}\left(\frac{P_{L}}{\bar{E}}\right)=\frac{-C_{1}^{2}}{\bar{E}}
\end{aligned}
$$

The fact that every derivative can be cast in terms of the mesh Péclet number and $\bar{E}$ facilitates the evaluation of $I G E_{01}$ and $I L E_{01}$. The Péclet number is available at every node and $\bar{E}(0)=\exp -\bar{P}_{L}$ is easily calculated if $\bar{P}_{L}$ is approximated with Hermite splines of the same degree. Note that $\bar{E}(1)=1$. As an example, if we use cubic Hermite splines the coefficients in Eqn 28 are

$$
\begin{aligned}
I L E_{01}= & \frac{1}{P_{L 0}}\left(\frac{1}{2}\left(P_{L 0} \exp \int_{0}^{1} P_{L} d \widehat{x}+P_{L 1}\right)+\frac{C_{1}}{12}\left(\exp \int_{0}^{1} P_{L} d \widehat{x}-1\right)\right) \\
I G E_{01}= & \frac{1}{2}\left(\exp \int_{0}^{1} P_{L} d \widehat{x}+1\right)-\frac{1}{12}\left(P_{L 0} \exp \int_{0}^{1} P_{L} d \widehat{x}-P_{L 1}\right) \\
& \int_{0}^{1} P_{L} d \widehat{x}=\frac{1}{2}\left(P_{L 0}+P_{L 1}\right)+\frac{1}{12}\left(P_{L 0}^{2}-P_{L 1}^{2}\right) \\
& C_{1}=\frac{P_{L}^{(\Delta v)}-P_{L 0}^{2} I L E_{01}}{I G E_{01}}
\end{aligned}
$$

As we are dealing with a nonlinear equation an iterative process is required. At the beginning of each iteration $C_{1}$ is first calculated and then $I L E_{01}, I G E_{01}$ 
and $\widetilde{k}$ are updated in order to evaluate $v$ at the nodes with the algebraic equation. The iterations are repeated till convergence.

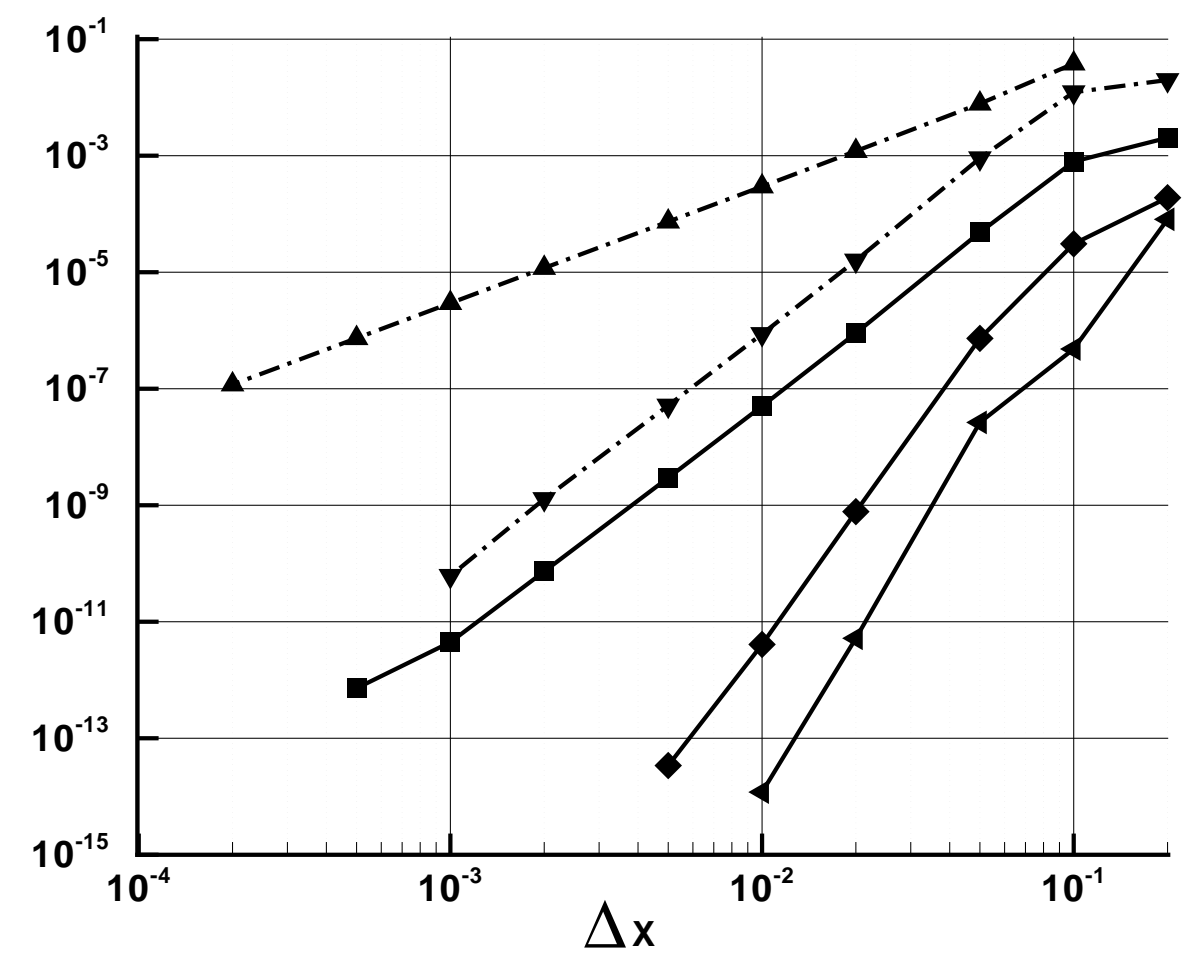

Fig. 10. Residuals for $\mu=0.04$. Solid lines correspond to Hermite splines and dash-dotted lines to finite-volume or finite-difference schemes explained in the text Cubic Hermite Quintic Hermite $\triangleleft$ Septic Hermite $\boldsymbol{\Delta}$ Central differencing $\mathbf{\nabla}$ Compact scheme

Some convergence problems were initially found for some Hermite splines and/or $\mu$ values but they were solved by starting the calculation with an initial estimation. This was obtained with the assumption that $P_{L}$ was constant over the interval and equal to $\bar{P}_{L}$, the latter calculated via Hermite splines. With this assumption

$$
I G E_{01}=\frac{1}{\bar{P}_{L}}\left(\exp \bar{P}_{L}-1\right) \quad ; \quad I L E_{01}=\frac{1}{P_{L 0}}\left(\exp \bar{P}_{L}-1\right)
$$


After this initial estimation the iterative process seemed to work fine.

In Fig. 10 the residuals for the case $\mu=0.04$ are shown. This particular value of $\mu$ was chosen because the solution contains a relatively broad region of large first and second derivatives. If, for instance, $\mu$ is increased to 0.1 the solution is very smooth for the whole domain and the case is not as attractive. The smaller $\mu$ the thinner is the boundary layer present near $x=1$, so $\mu$ was decreased to 0.01 for the second case tested.

As seen in the figure the computed results are excellent. The $L_{2}$ norm of the error with septic Hermite spline is $4.8110^{-7}$ with 10 nodes and reaches machine accuracy with 100 nodes $\left(1.1910^{-14}\right)$. Cubic Hermite spline is fourth-order. Quintic is slightly better than sixth-order and septic Hermite is slightly worse than eighth-order. The second-order scheme CDS and the compact scheme are shown for comparison.

In Fig. 11 the more stringent case of $\mu=0.01$ is portrayed. As expected the residuals for the initial $\Delta x$ are higher and the rates of convergence are less than those of the previous case with $\mu=0.04$. For some Hermite splines the program did not converge for large $\Delta x$ even after applying the initial estimation mentioned above and consequently there are points missing in the figure. With 100 intervals none of the schemes have reached machine accuracy but, for instance, the $L_{2}$ norm of the error with septic Hermite almost reaches machine accuracy with 500 intervals, $L_{2}=2.8410^{-13}$. Cubic Hermite convergence is fourth-order as CS, quintic Hermite is sixth-order and septic Hermite is eighth-order in a short $\Delta x$ interval. 


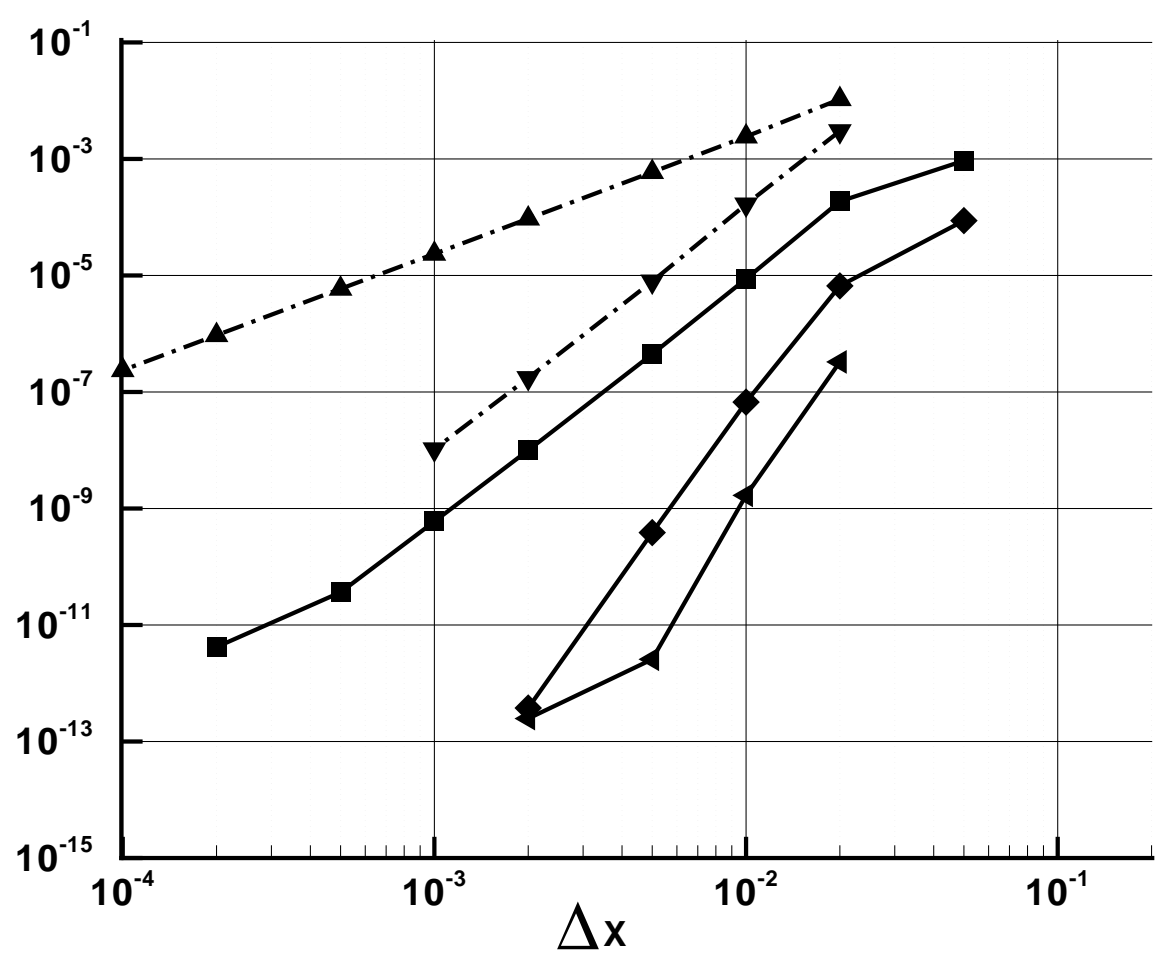

Fig. 11. Residuals for $\mu=0.01$ Same symbols as Fig. 10

\section{Discussion}

This new way of obtaining the algebraic equation that links the nodal values in a discretized domain seems to provide notable accuracy in all cases presented. The fact that the coefficients are exact makes the accuracy exclusively dependent on a numerical integration problem, which opens up the discretization problem to new approaches.

Although the source is relatively complicated, the first case is not very demanding as the diffusion coefficient only depends linearly on $x$, and $\rho v$ is constant. The algebraic coefficients can be accurately calculated because $I G E_{01}=$ $I L E_{01}$ and $P_{L}$ varies as $(1+x)^{-1}$, a function that behaves smoothly in the domain allowing good interpolation. Thus, it seems reasonable to find that 
it is the case where the highest accuracy has been attained. As expected the rate of convergence decreases as either the Péclet number increases or the order of the spline/quadrature is decreased. Results can always reach machine accuracy with a reasonable number of mesh points, especially for high spline orders.

It is worth commenting again that the algebraic coefficients given in Eq. 28 are exact and, hence, the solution may be obtained by analytically calculating the integrals and then solving the tridiagonal system of equations. This is a general characteristic, if the problem has an exact solution the integrals can be calculated analytically and viceversa, if the integrals allow an analytical primitive the PDE has an exact solution using Eq. 28, for arbitrary coefficients and source. In this test case the exact solution was in fact obtained following this route, although the results presented in this paper were always computed via numerical integration of the algebraic coefficients. When using the traditional discretization of the derivatives we can never end up with an exact expression for a three point stencil.

The second test case does not have an analytical solution. In order to calculate the error norms a reference solution that had been numerically obtained with a rather fine grid was employed. There is some uncertainty as to which numerical solution should be chosen to compare with. The septic Hermite spline solution was picked up based on previous experience in other test cases but there is no fundamental reason why the best approach should be the same in all cases. This issue warrants further work.

This approach seems to work well even when the boundary conditions are of von Newmann type. The exact algebraic solution allows the boundary value 
to be calculated in terms of the last internal node without having to adopt the usual strategy of assigning the same value. The handling of derivative boundary conditions is easy and not limited to zero gradient.

In the nonlinear equation the transformed exponential is adequate for moderate/high mesh Péclet. The expression that provides the values of $I L E_{01} / I G E_{01}$ for an n-ic Hermite spline is

$$
\frac{I L E_{01}}{I G E_{01}}=\frac{\left.\sum_{j=0}^{n} a_{j} \frac{j !}{\bar{P}_{L}^{j}}\right|_{I L E}}{\left.\sum_{j=0}^{n} a_{j} \frac{j !}{\bar{P}_{L}^{j}}\right|_{I G E}}
$$

as the factor containing $\exp \bar{P}_{L}$ dominates. When using the transformed exponential the $a_{j}$ coefficients are of the order of $\left(P_{L 0}-\bar{P}_{L}\right)^{j} / j$ ! so all terms in the sum are roughly of the same order, obviously depending on the actual values of Péclet. This is not true if the velocity field is prescribed as the importance of each $j$ term will depend on the particular $v$ field. In the case of the nonlinear equation, for which the velocity derivatives are calculated according to the appendix, it just happens that all terms are of the same importance, especially in the region near $x=1$ where $P_{L 0} / \bar{P}_{L}-1$ is most significant. We think that this is one of the reasons why the accuracy of this approach is so high for this nonlinear equation even for large $\Delta x\left(L_{2}=8.0910^{-5}\right.$ for $\mu=0.04$ and four! internal nodes with septic Hermite). In the integrals all solution derivatives affect the coefficients and the solution affects all derivatives.

Although this route has not been pursued in this work one obvious improvement is to work with adaptive meshes, with the location of nodes being related to an equidistribution of a predefined monitor function. The algebraic expression given in Eq. 28 is exact independently of the nodal distribution which can 
be modified arbitrarily by the numerical procedure according to the monitor function. As we have to calculate integrals in the intervals the global accuracy will hinge on how well these are calculated which in turn will depend on how well the integrand is interpolated. Thus, the monitor function should be related to a measure of the interpolation accuracy.

For two and three dimensions the transport equation along one coordinate contains the derivatives with respect to the other coordinates as source terms even if the whole equation is homogeneous. For instance, the 2D homogeneous transport equation

$$
\frac{\partial}{\partial x}\left(\rho u \phi-\Gamma \frac{\partial \phi}{\partial x}\right)+\frac{\partial}{\partial y}\left(\rho v \phi-\Gamma \frac{\partial \phi}{\partial y}\right)=0
$$

may be split in two

$$
\begin{aligned}
\frac{\partial}{\partial x}\left(\rho u \phi-\Gamma \frac{\partial \phi}{\partial x}\right) & =-\frac{\partial}{\partial y}\left(\rho v \phi-\Gamma \frac{\partial \phi}{\partial y}\right) \\
\frac{\partial}{\partial y}\left(\rho v \phi-\Gamma \frac{\partial \phi}{\partial y}\right) & =-\frac{\partial}{\partial x}\left(\rho u \phi-\Gamma \frac{\partial \phi}{\partial x}\right)
\end{aligned}
$$

Both equations can be discretized separately and then added up. The final discretized equation will be

$$
\begin{aligned}
& {\left[(\rho u)_{W} \widetilde{k}_{W P}+(\rho u)_{P}\left(\widetilde{k}_{P E}+\left.\frac{I L E_{01}}{I G E_{01}}\right|_{P E}\right)\right.} \\
& \left.+(\rho v)_{S} \widetilde{k}_{S P}+(\rho v)_{P}\left(\widetilde{k}_{P N}+\left.\frac{I L E_{01}}{I G E_{01}}\right|_{P N}\right)\right] \phi_{P}= \\
& (\rho u)_{W}\left(\widetilde{k}_{W P}+\left.\frac{I L E_{01}}{I G E_{01}}\right|_{W P}\right) \phi_{W}+(\rho u)_{P} \widetilde{k}_{P E} \phi_{E} \\
& +(\rho v)_{S}\left(\widetilde{k}_{S P}+\left.\frac{I L E_{01}}{I G E_{01}}\right|_{S P}\right) \phi_{S}+(\rho v)_{P} \widetilde{k}_{P N} \phi_{N} \\
& +\left.I S_{01}\right|_{W P}+\left.I S_{01}\right|_{S P} \\
& +\left(\left.\frac{I S G E_{01}}{I G E_{01}}\right|_{P E}-\left.\frac{I S G E_{01}}{I G E_{01}}\right|_{W P}\right)
\end{aligned}
$$




$$
+\left(\left.\frac{I S G E_{01}}{I G E_{01}}\right|_{P N}-\left.\frac{I S G E_{01}}{I G E_{01}}\right|_{S P}\right)
$$

The source integrals are

$$
\begin{aligned}
& \left.I S_{01}\right|_{W P}=L \int_{0}^{1} S(\widehat{x}) d \widehat{x}=\int_{W}^{P}-\frac{\partial}{\partial y}\left(\rho v \phi-\Gamma \frac{\partial \phi}{\partial y}\right) d x \\
& \left.I S_{01}\right|_{S P}=L \int_{0}^{1} S(\widehat{x}) d \widehat{x}=\int_{S}^{P}-\frac{\partial}{\partial x}\left(\rho u \phi-\Gamma \frac{\partial \phi}{\partial x}\right) d y
\end{aligned}
$$

The source integral in the ISGE terms can be obtained likewise. As seen in the last expression the calculation of a multidimensional transport equation involves the interpolation of some derivatives of the solution in order to evaluate the associated integrals. Work is underway to extend the ENATE approach to multidimensional problems.

\section{Conclusion}

A new way of obtaining the algebraic equation that links the nodal values of a transport variable has been proposed in this work. The coefficients are calculated based on the exact solution of the first-order ODE derived from the original second-order ODE and they involve the evaluation of integrals whose integrand is some function of the transport coefficients variation across the intervals. The algebraic equation also contains some source integrals that are exact if analytical primitives exist. This will not be the case in a general transport equation but the accuracy of source evaluation is similar to that of the coefficients if appropriate interpolating functions are employed. There is no limitation in the size ratio between two consecutive intervals which allows the use of arbitrary adaptive meshes. The accuracy of this approach for a 
three-point stencil is much higher than other traditional schemes, reaching eight order with septic Hermite spline.

\section{A Appendices}

\section{A.1 Derivation of the algebraic relation between nodes}

For completeness the normalized homogeneous solution is rewritten

$$
\begin{aligned}
\bar{\phi}^{N}(\widehat{x}) & =\bar{E}(\widehat{x})\left[\int_{0}^{\widehat{x}} \frac{d \widehat{x}^{\prime}}{\widehat{\Gamma} \bar{E}} / \int_{0}^{1} \frac{d \widehat{x}^{\prime}}{\widehat{\Gamma} \bar{E}}+\right. \\
& \left.+P_{L 0} \Phi \int_{0}^{1} \frac{\widehat{\lambda}}{\bar{E}} d \widehat{x}^{\prime}\left[\int_{0}^{\widehat{x}} \widehat{\hat{\lambda}} \overline{\bar{E}} d \widehat{x}^{\prime} / \int_{0}^{1} \frac{\widehat{\lambda}}{\bar{E}} d \widehat{x}^{\prime}-\int_{0}^{\widehat{x}} \frac{d \widehat{x}^{\prime}}{\widehat{\Gamma} \bar{E}} / \int_{0}^{1} \frac{d \widehat{x}^{\prime}}{\widehat{\Gamma} \bar{E}}\right]\right] \\
\Phi & =\frac{1}{1-F(1)} \frac{\phi_{l b}}{\Delta \phi}
\end{aligned}
$$

From now on a simplified notation will be used by defining $I L E_{0 \widehat{x}}$ and $I G E_{0 \widehat{x}}$.

$$
I L E_{0 \widehat{x}}=\int_{0}^{\widehat{x}} \frac{\widehat{\lambda}}{\bar{E}} d \widehat{x}^{\prime} \quad ; \quad I G E_{0 \widehat{x}}=\int_{0}^{\widehat{x}} \frac{d \widehat{x}^{\prime}}{\widehat{\Gamma} \bar{E}}
$$

$\mathrm{ILE}_{0 \widehat{x}}$ stands for 'Integral of Lambda E between 0 and $\widehat{x}$ ' and $\mathrm{IGE}_{0 \widehat{x}}$ for 'Integral of Gamma E between 0 and $\widehat{x}$. With this notation the solution is

$$
\bar{\phi}^{N}(\widehat{x})=\bar{E}(\widehat{x})\left[\frac{I G E_{0 \widehat{x}}}{I G E_{01}}+P_{L 0} \Phi I L E_{01}\left[\frac{I L E_{0 \widehat{x}}}{I L E_{01}}-\frac{I G E_{0 \widehat{x}}}{I G E_{01}}\right]\right]
$$

and its derivative is calculated as

$$
\frac{d \bar{\phi}^{N}}{d \widehat{x}}=P_{L}(\widehat{x}) \bar{\phi}^{N}(\widehat{x})+\frac{1}{\widehat{\Gamma}(\widehat{x}) I G E_{01}}+P_{L}(\widehat{x}) \Phi\left[1-\frac{1}{\widehat{\rho v}(\widehat{x})} \frac{I L E_{01}}{I G E_{01}}\right]
$$


where use has been made of $d \bar{E} / d \widehat{x}=P_{L} \bar{E}$. The factor that appears in the expression for the diffusive flux continuity at $\mathrm{P}$ is

$$
\frac{1}{P_{L}} \frac{d \bar{\phi}^{N}}{d \widehat{x}}=\phi^{N}(\widehat{x})+\frac{1}{P_{L 0} \widehat{\rho v}(\widehat{x}) I G E_{01}}+\Phi\left[1-\frac{1}{\widehat{\rho v}(\widehat{x})} \frac{I L E_{01}}{I G E_{01}}\right]
$$

Note that $P_{L} \widehat{\Gamma}=P_{L 0} \widehat{\rho v}$. Finally,

$$
\begin{aligned}
& \left.\frac{1}{P_{L}} \frac{d \bar{\phi}^{N}}{d \widehat{x}}\right|_{\widehat{x}=0}=\widetilde{k}+\Phi\left(1-\frac{I L E_{01}}{I G E_{01}}\right) \quad ; \quad \widetilde{k}=\frac{1}{P_{L 0} I G E_{01}} \\
& \left.\frac{1}{P_{L}} \frac{d \bar{\phi}^{N}}{d \widehat{x}}\right|_{\widehat{x}=1}=1+\frac{\widetilde{k}}{\widehat{\rho v}(1)}+\Phi\left(1-\frac{I L E_{01}}{\widehat{\rho v}(1) I G E_{01}}\right)
\end{aligned}
$$

With the new notation the particular solution is

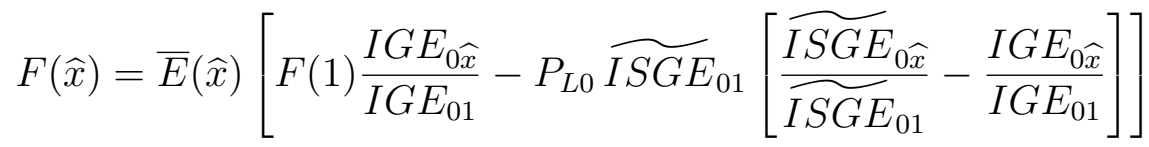

where $\widetilde{I S G E}$, 'Integral of Source Gamma E', is

$$
\widetilde{I S G E}_{0 \widehat{x}}=\int_{0}^{\widehat{x}} \frac{\widetilde{I S}}{\widehat{\Gamma} \widehat{\Gamma}^{\prime}} d \widehat{x}^{\prime}=\int_{0}^{\widehat{x}} \frac{\int_{0}^{\widehat{x}^{\prime}} \Pi_{s}\left(\widehat{x}^{\prime \prime}\right) d \widehat{x}^{\prime \prime}}{\widehat{\Gamma} \bar{E}} d \widehat{x}^{\prime}
$$

The factor that has to be calculated at the edges of the interval is

$$
\frac{1}{P_{L}} \frac{d F}{d \widehat{x}}=F(\widehat{x})+\frac{1}{P_{l 0}} \frac{F(1)}{\widehat{\rho v}(\widehat{x}) I G E_{01}}-\frac{1}{\widehat{\rho v}(\widehat{x})}\left[\widetilde{I S}_{0 \widehat{x}}-\frac{\widetilde{I S G E_{01}}}{I G E_{01}}\right]
$$

and finally

$$
\begin{aligned}
&\left.\frac{1}{P_{L}} \frac{d F}{d \widehat{x}}\right|_{\widehat{x}=0}=F(1) \widetilde{k}+{\widetilde{I S G E_{01}}}_{I G E_{01}} \\
&\left.\frac{1}{P_{L}} \frac{d F}{d \widehat{x}}\right|_{\widehat{x}=1}=F(1)+\frac{F(1)}{\widehat{\rho v}(1)} \widetilde{k}-\frac{1}{\widehat{\rho v}(1)}\left[\widetilde{I S}_{01}-\frac{\widehat{I S G E_{01}}}{I G E_{01}}\right]
\end{aligned}
$$

where 


$$
\widetilde{I S}_{01}=\int_{0}^{1} \Pi_{s}(\widehat{x}) d \widehat{x}
$$

Inserting these values in the diffusive flux continuity expression at $\mathrm{P}$ gives

$$
\begin{aligned}
& {\left[(\rho v)_{W} \widetilde{k}_{W P}+(\rho v)_{P}\left(\widetilde{k}_{P E}+\left.\frac{I L E_{01}}{I G E_{01}}\right|_{P E}\right)\right] \phi_{P}=} \\
& (\rho v)_{W}\left(\widetilde{k}_{W P}+\left.\frac{I L E_{01}}{I G E_{01}}\right|_{W P}\right) \phi_{W}+(\rho v)_{P} \widetilde{k}_{P E} \phi_{E} \\
& +\left.I S_{01}\right|_{W P} \\
& +\left(\left.\frac{I S G E_{01}}{I G E_{01}}\right|_{P E}-\left.\frac{I S G E_{01}}{I G E_{01}}\right|_{W P}\right)
\end{aligned}
$$

Note that the source-dependent terms are dimensional and they are related to those in previous expressions by

$$
\begin{aligned}
& I S G E_{01}=(\rho v)_{l b} \Delta \phi \widetilde{I S G E}_{01}=\int_{0}^{1} \frac{I S_{0 \widehat{x}^{\prime}}}{\widehat{\Gamma} \bar{E}} d \widehat{x}^{\prime}=\int_{0}^{1} \frac{L \int_{0}^{x^{\prime}} S\left(\widehat{x}^{\prime \prime}\right) d \widehat{x}^{\prime \prime}}{\widehat{\Gamma} \bar{E}} d \widehat{x}^{\prime} \\
& I S_{01}=(\rho v)_{l b} \Delta \phi \widetilde{I S}_{01}=L \int_{0}^{1} S(\widehat{x}) d \widehat{x}=\int_{x_{l b}}^{x_{r b}} S(x) d x
\end{aligned}
$$

\section{A.2 Velocity derivatives}

To determine the integral $\int_{0}^{1} P_{L}^{(\phi)} d \widehat{x}^{\prime}$ it is necessary to provide $P_{L}^{(\phi)}$ and its derivatives at 0 and 1 . These values are also used in the calculation of the other integrals involved. If $v$ is calculated with its transport (Navier-Stokes) equation, the solution in a generic interval is that developed in this paper and its derivative is

$$
\frac{d \widehat{v}}{d \widehat{x}}=\frac{d F}{d \widehat{x}}+(1-F(1)) \frac{d \bar{v}^{N}}{d \widehat{x}}
$$

With a bit of algebra its first and second derivatives can be obtained at $\widehat{x}$ as 


$$
\begin{aligned}
\frac{d \widehat{v}}{d \widehat{x}}= & P_{L}^{(v)} \frac{v}{\Delta v}+\frac{1}{\widehat{\mu}} \frac{1+P_{L 0}^{(v)}\left({\widetilde{I S G E_{01}}}-\frac{v_{l b}}{\Delta v} I L E_{01}\right)}{I G E_{01}}-\frac{P_{L 0}^{(v)}}{\widehat{\mu}} \int_{0}^{\widehat{x}} \Pi_{s} d \widehat{x}^{\prime} \\
\frac{d^{2} \widehat{v}}{d \widehat{x}^{2}}= & \frac{1}{\Delta v} \frac{d}{d \widehat{x}}\left(P_{L}^{(v)} v\right)+\frac{d}{d \widehat{x}}\left(\frac{1}{\widehat{\mu}}\right) \frac{1+P_{L 0}^{(v)}\left({\widetilde{I S G E_{01}}}-\frac{v_{l b}}{\Delta v} I L E_{01}\right)}{I G E_{01}} \\
- & P_{L 0}^{(v)} \frac{d}{d \widehat{x}}\left(\frac{1}{\widehat{\mu}} \int_{0}^{\widehat{x}} \Pi_{s} d \widehat{x}^{\prime}\right) \\
& P_{L 0}^{(v)}=\frac{(\rho v)_{l b} L}{\mu_{l b}} ; \quad P_{L}^{(v)}=\frac{\rho v L}{\mu} ; \Delta v=v_{r b}-v_{l b} \\
& \widehat{I S G E}_{01}=\int_{0}^{1} \frac{\int_{0}^{\widehat{x}} \Pi_{s} d \widehat{x}^{\prime}}{\widehat{\Gamma} \bar{E}} d \widehat{x}
\end{aligned}
$$

the third derivative being obvious. Using these derivatives we can deduce those of $P_{L}^{(\phi)}$. For instance, for a constant density case, the derivative of the mesh Péclet number associated to a generic variable $\phi$ with diffusion coefficient $\Gamma$ can be obtained as

$$
\frac{d P_{L}^{(\phi)}}{d \widehat{x}}=\frac{\mu}{\Gamma} P_{L}^{(\Delta v)} \frac{d \widehat{v}}{d \widehat{x}}+P_{L}^{(v)} \mu \frac{d}{d \widehat{x}}\left(\frac{1}{\Gamma}\right) \quad ; \quad P_{L}^{(\Delta v)}=\frac{\rho \Delta v L}{\mu}
$$

in terms of velocity derivatives. Similarly for the second and third derivatives. The second term is negligible if the variation of $\Gamma$ in the domain is much less than that of $v$.

\section{References}

[1] Wong HH, Raithby GD, Improved finite difference methods based on a critical evaluation on the approximation errors. Numer. Heat Transfer 1979;2:139-163

[2] Allen DN de G, Southwell RV, Relaxation methods applied to determine the motion in 2 dimensions of a viscous fluid past a fixed cylinder. Q. J. Mech. Appl. Math. 1955;8:129:145 
[3] Thiart GD. Finite difference scheme for the numerical solution of fluid flow and heat transfer problems on nonstaggered grids. Numer. Heat Transfer, part B 1990;17: 43-62

[4] Thiart GD. Improved finite difference scheme for the solution of convectiondiffusion problems with the SIMPLEN algorithm. Numer. Heat Transfer, part B 1990;18: 81-95

[5] Harms TM, von Backström TW, du Plessis JP. Reformulation of the SIMPLEN discretization scheme to accomodate noncentralized interfaces. Numer. Heat Transfer, part B 1991;20:127-144

[6] Wang GW, Wei JG, Tao WQ, An improved numerical algorithm for solution of convective heat transfer problems on nonstaggered grid system. Heat Mass Transfer 1998;33:273-280

[7] Sakai, K. Locally Exact Numerical Scheme for Transport Equations with Source Terms-LENS. J. of Nuclear Sci. and Tech., 1992;29:824-827

[8] Günther G. Conservative versions of locally exact consistent upwind scheme of second order (LECUSSO-SCHEME). Int. J. Numer. Methods Eng. 1992;34:793800

[9] Sakai K. Optimized locally exact numerical scheme based on finite variable difference method and characteristic polynomial analysis method. J. of Nuclear Sci. and Tech., 1995;32:42-49

[10] Sakai K. A new finite variable difference method with application to locally exact numerical scheme. J. of Comp. Phys. 1996;124:301-308

[11] Sakai K., Zhang GS. A numerical scheme for transport equations with spatially distributed coefficients based on locally exact difference method. J. of Comp. Phys. 1997;134:332341 
[12] Kriventsev V., Ninokata H. An effective, locally exact finite-difference scheme for convection-diffusion problems. Numer. Heat Transfer, part B 1999;36:183-205

[13] Figueiredo JR, A unified finite-volume finite-differencing exponential-type convective-diffusive fluid transport equations. J. Braz. Soc. Mech. Sci. 1997;9:371391

[14] Figueiredo JR, Moraes de Oliveira JP. Comparative study of UNIFAES and other finite volume schemes for the discretization of advective and viscous fluxes in incompressible Navier-Stokes equations, using various mesh structures. Numer. Heat Transfer part B, 2009;55:379-405

[15] Sheu TW, Lin RK An incompressible Navier-Stokes model implemented on nonstaggered grid. Num. Heat Transfer B 2003;44:277-294

[16] Chen CJ, Naseri-Neshat H, Ho KS. Finite analytic numerical solution of heat transfer in two-dimensional cavity flow. Num. Heat Transfer 1981;4:179-197

[17] Chen CJ, Naseri-Neshat H, Ho KS. Development of finite analytic numerical method for unsteady two-dimensional Navier-Stokes equations. J. of Comp. Phys. $1984 ; 53: 209-226$

[18] Chen CJ, Bernatz R, Carlson KD, Lin W. Finite analytic method in flows and heat transfer. 2000 Taylor and Francis.

[19] Azmy YY. A nodal integral method for the numerical solution of incompressible fluid flow problems. 1982 Master's thesis. University of Illinois

[20] Rizwan-Uddin. A second-order space and time nodal method for the onedimensional convection-diffussion equation. Comp. and Fluids 1997;26:233247

[21] ten Thije Boonkamp JHM., Anthonissen MJH., The finite-volume complete-flux scheme for advection-diffusion-reaction equations. J. Sci. Comput. 2011;46:47-70 
[22] Pascau A. An exact discretization for a transport equation with piecewiseconstant coefficients and arbitrary source. Comp. and Fluids 2013;75:42-50

[23] Sen S. A new family of $(5,5)$ CC-4OC schemes applicable for unsteady NavierStokes equations J. of Comp. Phys. 2013;251:251-271

[24] Tian ZF., Dai SQ. High-order compact exponential finite difference methods for convection-diffusion type problems. J. of Comp. Phys. 2007;220:952-974

[25] Davis PJ, Interpolation and approximation. 1975. Dover publishers. 